\title{
HERDING, TREND CHASING, AND MARKET VOLATILITY
}

\author{
CORRADO DI GUILMI*, XUE-ZHONG HE** AND KAI LI** \\ *Economics Discipline Group, UTS Business School \\ **Finance Discipline Group, UTS Business School \\ University of Technology, Sydney \\ PO Box 123, Broadway, NSW 2007, Australia \\ corrado.diguilmi@uts.edu.au, tony.he1@uts.edu.au, kai.li@uts.edu.au
}

\begin{abstract}
We introduce a heterogeneous agent asset pricing model in continuoustime to show that, although trend chasing, switching and herding all contribute to market volatility in price and return and to volatility clustering, but their impacts are different. The fluctuations of the market price and return and the level of the significant autocorrelations (ACs) of the absolute and squared returns increase with herding and trend chasing based on long time horizon. However an increase in switching intensity reduces the return volatility and in particular low switching reduces the price volatility and increases the level of the significant ACs, but the effect becomes opposite when the switching intensity is high. We also show that market noise plays a more important role than fundamental noise on the power-law behavior of returns.
\end{abstract}

Key words: Heterogeneous beliefs, herding, switching, stability, volatility, stochastic delay differential equations.

JEL Classification: C62, D53, D84, G12

Date: April 16, 2014.

Acknowledgments: We would like to thank the participants to the 2013 Nonlinear Economic Dynamics Conference, Siena, July 2-4, 2013, a referee and the editors of the special issue for helpful comments. Financial support for He from the Australian Research Council (ARC) under Discovery Grant (DP130103210) is gratefully acknowledged. The usual caveats apply.

Corresponding author: Xue-Zhong (Tony) He, Finance Discipline Group, UTS Business School, University of Technology, Sydney, tony.he1@uts.edu.au. 


\section{INTRODUCTION}

Trend chasing, switching among different trading strategies and herding are the most commonly observed boundedly rational behaviors of investors in financial markets and this paper studies their impact on market volatility. Large fluctuations in market price, excess volatility in return, and volatility clustering are the most common stylized facts in financial markets. The question is how differently these boundedly rational behaviors of investors contribute to market volatility. This paper introduces a heterogeneous agent asset pricing model in a continuous-time framework to address this question. We show that herding and trend chasing based on long time horizon increase market volatility in price and return. However, the effect of switching is different for price volatility and becomes opposite for return volatility. We also show that these boundedly rational behavior of investors contribute to the power-law behavior, characterized by insignificant level of autocorrelations $(\mathrm{ACs})$ in the returns and significant and decaying ACs in the absolute and squared returns; however, their effects are different. More precisely, the levels of the significant ACs in return volatility increase with the herding and trend chasing based on long time horizon, but increase initially and then decrease as the switching intensity increases. In general, it is the interaction of nonlinear dynamics and noises that generates realistic market price dynamics. We show that the market noise plays a more important role than the fundamental noise in generating the power-law behavior. To our knowledge, this is the first paper showing that the herding and switching have opposite effect on the return volatility and different impact on the power-law behavior.

Over the last three decades, empirical evidence, unconvincing justifications of the assumption of unbounded rationality and the recognition of the relevance of investor psychology have led to the incorporation of heterogeneous and boundedly rational behavior of investors, such as trend chasing, switching and herding, into asset price and financial market modeling. Cross-sectional and time series momentums based on trend chasing behavior have been well documented in empirical literature (see Moskowitz et al. 2012 and the reference cited there), in which the time horizon used 
to calculate the trend plays a very important role in the mechanism generating momentum profitability (He and Li 2014 and He, Li and Li 2014). Adaptive switching of agents to better performed strategy can lead to market price fluctuations, bubbles and crashes (see for example, Brock and Hommes, 1997, 1998 in discrete-time and $\mathrm{He}$ and Li, 2012 in continuous-time). Herding refers broadly to the tendency of many different agents to take similar actions at roughly the same time. Scharfstein and Stein (1990) attribute it to the reputational concerns and the unpredictable components to investment outcomes. Banerjee (1992) shows that herd behavior is rational in term of obtaining others' information.

This paper is closely related to the recent development of heterogeneous agent models (HAMs) in considering financial markets as expectation feedback systems where asset price fluctuations can be caused by an endogenous mechanism with heterogeneity and bounded rationality. Various agent-based financial market models have been developed to incorporate trend chasing, switching and herding. For instance, by considering two types of traders, typically fundamentalists and trend followers, Beja and Goldman (1980) and Chiarella (1992) among many others have shown that interaction of agents with heterogeneous expectations may lead to market instability. More significantly, Brock and Hommes $(1997,1998)$ introduce the concept of an adaptively rational equilibrium in a discrete-time framework. Agents adapt their beliefs over time by choosing from different predictors or expectation functions based upon their past performance (such as realized profits). Such boundedly rational behavior of agents can also lead to market instability. More recently, these models have been extended to a continuous-time framework (see He et al. (2009), He and Zheng (2010) and He and Li (2012)), which provides an uniformed approach in dealing with the effect of time horizon used for trend chasing. Within a continuous-time framework, Lux (1995) and Alfarano et al. (2008) model the herding behavior through the master equation and show that herding can give rise to realistic time series. Within a discrete-time framework, Hohnisch and Westerhoff (2008) find that herding behavior at the level of individual economic sentiment may lead to enduring business cycles, while Franke and Westerhoff (2012) show a strong role for the herding component in generating realistic moments in financial time 
series. Overall, these models have successfully explained several market features (such as market booms and crashes, deviations of the market price from the fundamental price), the stylized facts (such as skewness, kurtosis, volatility clustering and fat tails of returns) and the power-law behavior. We refer the reader to Hommes (2006), LeBaron (2006), Chiarella et al. (2009), Lux (2009), and Chen et al. (2012) for surveys of the recent development in this literature.

This paper provides a unified framework in a continuous-time model to examine the joint impact of trend chasing, switching and herding on the market price dynamics and to compare different roles they play in generating market volatility in price and return and the power-law behavior of stock return volatility. We consider a continuous-time financial market with two types of agents: the fundamentalists who trade on the fundamental value and the trend followers who extrapolate the market price trend based on a weighted moving average price over a finite time horizon. The herding behavior among the agents is characterized by the master equation. The market price is determined by a market maker who adjusts the market price to the excess demand from the fundamentalists and trend followers, together with a noisy demand. The continuous-time setup chosen in the paper not only mathematically facilitates modeling the time horizon of the historical price information used by the trend followers, but also easily accommodates the herding behavior through the master equation with endogenously determined volatility.

We first examine the dynamics of the underlying deterministic model. Differently from the adaptive switching model in He and Li (2012), we find that the herding mechanism does not affect the local stability of the steady state fundamental price, although it does affect the nonlinear behavior. Based on the analysis of the deterministic dynamics, we then study the joint impact of trend chasing, herding and switching on the volatility of the market price and returns of the stochastic model. We find that the trend chasing based on historical prices over a long time horizon always leads to high volatility in both market prices and stock returns, which is characterized by the destabilizing effect of the trend chasing. Also, herding and switching have very different effect on the market volatility. A strong herding contributes to high fluctuation in market fractions and market price and hence generates 
high volatilities in prices and returns, while a more intense switching reduces the return volatility and has a non-monotonic effect on the volatilities of market fractions and prices. More interestingly, we observe a "hump" shaped volatility in the market fraction and an "U"-shaped price volatility as the switching intensity increases.

We explore further the potential of the model to generate the power-law behavior in volatility by examining the impact of the different noises (including the fundamental noise, the market noise and the market fraction noise), time horizon, switching and herding on the level of ACs of the returns, absolute returns, and squared returns. We find that market noise is the main driving force in generating the power-law behavior. The levels of the AC patterns become more significant as the time horizon and herding increase, but non-monotonically with the switching. Specifically, an initial increase in the switching intensity leads to an increase in the significant levels of ACs, but the ACs decrease as the switching increases further. In general, it is the combination of switching and herding, together with the market noise, that generates realistic power-law behavior.

The paper is organized as follows. We first introduce a stochastic HAM of asset pricing in continuous-time with trend chasing, herding, switching and heterogeneous beliefs in Section 2. In Section 3, we apply stability and bifurcation theory of delay differential equations, together with numerical analysis of the nonlinear system, to examine the impact of herding, switching and time horizon used by the trend followers on market stability. The effect of and different roles played by trend chasing, herding and switching on the market volatility and power-law behavior are then discussed in Sections 4 and 5, respectively. Section 6 concludes. All proofs and some additional results are given in the Appendices.

\section{The Model}

Consider a financial market with a risky asset (such as stock market index) and let $P(t)$ be the (cum dividend) price of the risky asset at time $t$. Following the standard approach of HAMs (see, for example, Brock and Hommes (1998)), we assume that the market consists of fundamentalists, who trade according to the fundamental value of the risky asset, trend followers, who trade based on price 
trend of a weighted moving averages of historical prices over a time horizon, and a market maker, who clears the market by providing liquidity. The behavior of the fundamentalists and trend followers is modeled as usual. Different from the discretetime HAMs in the literature (for example, Chiarella and He, 2002 and 2003), we consider a continuous-time setup to accommodate different time horizon used by the trend followers and the stochastic master equation characterizing the herding behavior of agents. For completeness, we introduce the demand functions of the fundamentalists and the trend followers briefly and refer the reader to $\mathrm{He}$ and $\mathrm{Li}$ (2012) (HL model hereafter) for details.

The fundamentalists believe that the market price $P(t)$ is mean-reverting to the fundamental value $F(t)$ that can be estimated based on fundamental analysis. They buy (sell) the stock when the current price $P(t)$ is below (above) the fundamental value $F(t)$. For simplicity, we assume that the demand of the fundamentalists, $Z_{f}(t)$ at time $t$, is proportional to the deviation of the market price $P(t)$ from the fundamental value $F(t)$, namely,

$$
Z_{f}(t)=\beta_{f}[F(t)-P(t)]
$$

where $\beta_{f}>0$ is a constant, measuring the speed of mean-reversion of the market price to the fundamental value, weighted by the risk tolerance of the fundamentalists. To focus on the price dynamics, we simply assume that the fundamental value follows a stochastic process 1

$$
d F(t)=\frac{1}{2} \sigma_{F}^{2} F(t) d t+\sigma_{F} F(t) d W_{F}(t), F(0)=\bar{F},
$$

\footnotetext{
${ }^{1}$ It follows from Eq. (2.2) that the fundamental return defined by $d(\ln (F(t)))=\sigma_{F} d W_{F}(t)$ is a pure white noise process following a normal distribution with mean of 0 and standard deviation of $\sigma_{F} \sqrt{d t}$. This implies that any non-normality and volatility clustering of market returns that generated by the model are not carried from the fundamental returns. In the present treatment we use the zero-mean fundamental return process (2.2). Suggested by the referee, we have also investigated the case of positive drift in the fundamental return process: $d(\ln F(t))=\mu_{F} d t+$ $\sigma_{F} d W_{F}(t)$ with $\mu_{F}=c \sigma_{F}^{2}>0$ for a constant $c$. Numerical simulations show this does not alter the effects of time horizon, herding and switching on the volatilities and autocorrelations documented in Sections 4 and 5, although an increase in $\mu_{F}$ reduces the level of return volatility and makes the autocorrelations decay slower.
} 
where $\sigma_{F}>0$ represents the volatility of the fundamental return and $W_{F}(t)$ is a standard Wiener process.

The trend followers believe that the future market price follows a price trend $u(t)$. When the current price is above (below) the trend, the trend followers believe that the price will rise (fall) and hold a long (short) position of the risky asset. We assume that the demand of the trend followers is given by

$$
Z_{c}(t)=\tanh \left[\beta_{c}(P(t)-u(t))\right]
$$

The $S$-shaped hyperbolic demand function capturing the trend following behavior is well documented in the HAM literature (see, for example, Chiarella et al. 2009), where the parameter $\beta_{c}$ represents the extrapolation rate of the trend followers on the future price trend. The limited position may reflect the wealth constraint or the cautiousness of the trend followers when the price deviates from the trend significantly2. Among various estimators of the price trend used in practice, we assume that the price trend $u(t)$ at time $t$ is calculated by an exponentially decaying weighted average of historical prices over a time horizon $[t-\tau, t]$,

$$
u(t)=\frac{k}{1-e^{-k \tau}} \int_{t-\tau}^{t} e^{-k(t-s)} P(s) d s,
$$

where time delay $\tau \in[0, \infty)$ represents a time horizon used to calculate the price trend and $k>0$ is a decay rate. Equation (2.4) implies that, when forming the price trend, the trend followers believe that the more recent prices contain more information about the future price movement so that the weights associated to the historical prices decay exponentially. In particular, when $k \rightarrow 0$, the price trend $u(t)$ in equation (2.4) is simply given by the standard moving average with equal weights,

$$
u(t)=\frac{1}{\tau} \int_{t-\tau}^{t} P(s) d s
$$

\footnotetext{
${ }^{2}$ Alternative to (2.1), one can assume that the fundamentalists also take limited positions as the trend followers, $Z_{f}(t)=\tanh \left[\beta_{f}(F(t)-P(t))\right]$. Clearly this assumption does not affect the local stability of the fundamental price. Intuitively, because of the stabilizing role of the fundamentalists, this limited position will make the market price less stable. This is supported by numerical simulations (not reported here) showing that the fluctuations are amplified when the system become unstable.
} 
When $k \rightarrow \infty$, all the weights go to the current price so that $u(t) \rightarrow P(t)$. For the time delay, when $\tau \rightarrow 0$, the trend followers regard the current price as the price trend. When $\tau \rightarrow \infty$, they use all the historical prices to form the price trend

$$
u(t)=\frac{1}{k} \int_{-\infty}^{t} e^{-k(t-s)} P(s) d s .
$$

In general, for $0<k, \tau<\infty$, equation (2.4) can be expressed as a delay differential equation with time delay $\tau$,

$$
d u(t)=\frac{k}{1-e^{-k \tau}}\left[P(t)-e^{-k \tau} P(t-\tau)-\left(1-e^{-k \tau}\right) u(t)\right] d t .
$$

Let $N_{f}(t)$ and $N_{c}(t)$ be the numbers of the fundamentalists and trend followers, respectively, at time $t$, satisfying $N_{f}(t)+N_{c}(t)=N$, a constant. Denote by $n_{f}(t)=$ $N_{f}(t) / N$ and $n_{c}(t)=N_{c}(t) / N$ the market fractions of the fundamentalists and trend followers, respectively. The net profits of the fundamental and trend following strategies over a small time interval $[t, t+d t]$ are then measured by, respectively,

$$
\pi_{f}(t) d t=Z_{f}(t) d P(t)-C_{f} d t, \quad \pi_{c}(t) d t=Z_{c}(t) d P(t)-C_{c} d t
$$

where $C_{f}, C_{c} \geq 0$ are constant costs of the strategies per time unit. The performances of the strategies are measured by the cumulated and weighted net profits over time horizons $\left[t-\tau_{i}, t\right]^{3}$,

$$
U_{i}(t)=\frac{\eta_{i}}{1-e^{-\eta_{i} \tau_{i}}} \int_{t-\tau_{i}}^{t} e^{-\eta_{i}(t-s)} \pi_{i}(s) d s, \quad i=f, c,
$$

where $\eta_{i}>0$ and $\tau_{i}>0$ for $i=f, c$ represent the decay parameter and time horizon used, respectively, to measure the performance of the fundamentalists and trend followers. Consequently,

$$
d U_{i}(t)=\eta_{i}\left[\frac{\pi_{i}(t)-e^{-\eta_{i} \tau_{i}} \pi_{i}\left(t-\tau_{i}\right)}{1-e^{-\eta_{i} \tau_{i}}}-U_{i}(t)\right] d t, \quad i=f, c .
$$

Denote by $a(t)$ the transition probability of an agent switching from trend follower to fundamentalist and by $b(t)$ the probability of the inverse transition. Following Lux (1995), the probabilities can be quantified by

$$
a(t)=v e^{\beta\left(U_{f}(t)-U_{c}(t)\right)}, \quad b(t)=v e^{\beta\left(U_{c}(t)-U_{f}(t)\right)},
$$

\footnotetext{
${ }^{3}$ The investment time horizon can be different for the fundamentalists and trend followers and therefore they may use different time horizon when evaluating the performance of their strategies.
} 
where $\beta$ measures the switching intensity and $v>0$ captures the intensity of herding (explained in the following). Let $\zeta(t)$ denote the transition rate of observing a change of an agent from trend follower to fundamentalist and $\xi(t)$ denote the transition rate of recording the opposite transition. Both $\zeta(t)$ and $\xi(t)$ are assumed to be proportional to the transition probability of the switching and the corresponding market fractions to capture the herding behavior. Then the transition rates can be expressed as

$$
\begin{aligned}
& \zeta(t)=\left(1-n_{f}(t)\right) a(t)=v\left(1-n_{f}(t)\right) e^{\beta\left(U_{f}(t)-U_{c}(t)\right)}, \\
& \xi(t)=n_{f}(t) b(t)=v n_{f}(t) e^{\beta\left(U_{c}(t)-U_{f}(t)\right)} .
\end{aligned}
$$

Note that, when $\beta=0$, a large $v$ means a strong herding among the agents. Hereafter, we use $\beta$ and $v$ to measure the (performance based) switching and herding, respectively, among the agents. Following Lux (1995), the master equation measuring the variation of probability in a unit of time by taking the number of fundamentalists as a state variable follows

$$
\frac{d p\left(N_{f}, t\right)}{d t}=\zeta(t) p\left(N_{f}-1, t\right)+\xi(t) p\left(N_{f}+1, t\right)-[\zeta(t)+\xi(t)] p\left(N_{f}, t\right),
$$

where $p\left(N_{f}, t\right)$ is the probability of recording a number of $N_{f}$ fundamentalists at time $t$. Following Chiarella and Di Guilmi (2011b), the dynamics of the population evolution can be characterized by 4

$$
d n_{f}(t)=n_{f}(t)\left[-(\zeta(t)+\xi(t)) n_{f}(t)+\zeta(t)\right] d t+\sigma_{n_{f}} d W_{n_{f}}(t)
$$

where

$$
\sigma_{n_{f}}(t)=\frac{\sqrt{\zeta(t) \xi(t)}}{\zeta(t)+\xi(t)}
$$

and $W_{n_{f}}(t)$ is the stochastic fluctuation component in the market population fraction of fundamentalists, which is assumed to be independent from the fundamental noises $W_{F}(t)$.

Finally, the price $P(t)$ at time $t$ is adjusted by the market maker according to the aggregate market excess demand, that is,

$$
d P(t)=\mu\left[n_{f}(t) Z_{f}(t)+n_{c}(t) Z_{c}(t)\right] d t+\sigma_{M} d W_{M}(t)
$$

\footnotetext{
${ }^{4}$ The derivation is given in Appendix $\mathrm{A}$
} 
where $\mu>0$ represents the speed of the price adjustment by the market maker, $\sigma_{M}>0$ is a constant and $W_{M}(t)$ is a standard Wiener process capturing the random excess demand process either driven by unexpected market news or noise traders 5 which is independent of $W_{F}(t)$ and $W_{n_{f}}(t)$.

To sum up, the market price of the risky asset is determined according to the following stochastic delay differential system with three different time delays and three noise processes

$$
\left\{\begin{aligned}
d P(t) & =\mu\left[n_{f}(t) Z_{f}(t)+\left(1-n_{f}(t)\right) Z_{c}(t)\right] d t+\sigma_{M} d W_{M}(t) \\
d u(t)= & \frac{k}{1-e^{-k \tau}}\left[P(t)-e^{-k \tau} P(t-\tau)-\left(1-e^{-k \tau}\right) u(t)\right] d t \\
d n_{f}(t)=v n_{f}(t)\left[\left(1-n_{f}(t)\right)^{2} e^{\beta\left(U_{f}(t)-U_{c}(t)\right)}-n_{f}^{2}(t) e^{\beta\left(U_{c}(t)-U_{f}(t)\right)}\right] d t & \quad+\sigma_{n_{f}} d W_{n_{f}}(t) \\
d U_{f}(t)= & \frac{\eta_{f}}{1-e^{-\eta_{f} \tau_{f}}}\left[\pi_{f}(t)-e^{-\eta_{f} \tau_{f}} \pi_{f}\left(t-\tau_{f}\right)-\left(1-e^{-\eta_{f} \tau_{f}}\right) U_{f}(t)\right] d t \\
d U_{c}(t)= & \frac{\eta_{c}}{1-e^{-\eta_{c} \tau_{c}}}\left[\pi_{c}(t)-e^{-\eta_{c} \tau_{c}} \pi_{c}\left(t-\tau_{c}\right)-\left(1-e^{-\eta_{c} \tau_{c}}\right) U_{c}(t)\right] d t \\
d F(t)= & \frac{1}{2} \sigma_{F}^{2} F(t) d t+\sigma_{F} F(t) d W_{F}(t)
\end{aligned}\right.
$$

where $Z_{f}(t)$ and $Z_{c}(t)$ are defined by (2.1) and (2.3), respectively, and $\pi_{i}(t)$ is defined by (2.8) for $i=f, c$. The stochastic differential system (2.17) characterizes the market price dynamics with heterogeneity in trading strategies, trend chasing, switching and herding. The main difference between the system (2.17) and the HL model is that the population evolution is characterized through the replicator dynamics in the HL model, instead of the master equation on herding in system (2.17) and therefore the dynamics of market fraction $n_{f}$ is specified with endogenously determined volatility. The other processes $\left(P, u, U_{f}, U_{c}, F\right)$ are the same for the two systems.

In the following sections, we first conduct a stability analysis of the underlying deterministic model. Then we examine the impact of the interaction of the deterministic dynamics with the noises on the fluctuations of the market population

\footnotetext{
${ }^{5}$ The additive noise comes naturally when a demand from noise traders is introduced into the aggregated excess demand function.
} 
fractions (of using different strategies) and market volatility in both prices and return. Furthermore, we explore the power-law behavior in volatility.

\section{The Stability Analysis of the Deterministic Model}

To understand the interaction of the nonlinear deterministic dynamics and the noise processes, we first study the local stability of the corresponding deterministic system. By assuming $\sigma_{M}=0$ and hence $F(t)=\bar{F}$ and considering the mean process of the market fraction of the fundamentalists, system (2.17) reduces to

$$
\left\{\begin{aligned}
\frac{d P(t)}{d t} & =\mu\left[n_{f}(t) \beta_{f}[F(t)-P(t)]+\left(1-n_{f}(t)\right) \tanh \left[\beta_{c}(P(t)-u(t))\right]\right. \\
\frac{d u(t)}{d t} & =\frac{k}{1-e^{-k \tau}}\left[P(t)-e^{-k \tau} P(t-\tau)-\left(1-e^{-k \tau}\right) u(t)\right] \\
\frac{d n_{f}(t)}{d t} & =v n_{f}(t)\left[\left(1-n_{f}(t)\right)^{2} e^{\beta\left(U_{f}(t)-U_{c}(t)\right)}-n_{f}^{2}(t) e^{\beta\left(U_{c}(t)-U_{f}(t)\right)}\right] \\
\frac{d U_{f}(t)}{d t} & =\frac{\eta_{f}}{1-e^{-\eta_{f} \tau_{f}}}\left[\pi_{f}(t)-e^{-\eta_{f} \tau_{f}} \pi_{f}\left(t-\tau_{f}\right)-\left(1-e^{-\eta_{f} \tau_{f}}\right) U_{f}(t)\right] \\
\frac{d U_{c}(t)}{d t} & =\frac{\eta_{c}}{1-e^{-\eta_{c} \tau_{c}}}\left[\pi_{c}(t)-e^{-\eta_{c} \tau_{c}} \pi_{c}\left(t-\tau_{c}\right)-\left(1-e^{-\eta_{c} \tau_{c}}\right) U_{c}(t)\right]
\end{aligned}\right.
$$

where

$$
\pi_{i}(t)=\mu Z_{i}(t)\left[n_{f}(t) Z_{f}(t)+\left(1-n_{f}(t)\right) Z_{c}(t)\right]-C_{i}, \quad i=f, c .
$$

The system has a steady state 6

$$
Q:=\left(P, u, n_{f}, U_{f}, U_{c}\right)=\left(\bar{F}, \bar{F}, \frac{1}{1+e^{\beta\left(C_{f}-C_{c}\right)}},-C_{f},-C_{c}\right),
$$

in which the market price is given by the fundamental value. We call $Q$ the fundamental steady state of the system (3.1). At the fundamental steady state, the market fraction of fundamentalists becomes $n_{f}^{*}=\frac{1}{1+e^{\beta\left(C_{f}-C_{c}\right)}}$. When $C_{f}=C_{c}$, $n_{f}^{*}=n_{c}^{*}=0.5$, meaning that the market fractions at the fundamental steady state is independent from the switching intensity $\beta$ and the herding parameter $v$. However, when the fundamental strategy costs more, that is $C_{f}>C_{c}$, then $n_{c}^{*}>n_{f}^{*}$, meaning

\footnotetext{
${ }^{6}$ In addition, the line $P=u, n_{f}=0, U_{f}=-C_{f}, U_{c}=-C_{c}$ is a steady state line of the system. This means that the system has infinite many steady states. Near the line, the solution with different initial values converge to different steady states on the line. Hence the line is locally attractive. A similar result is found in He et al. (2009) and He and Zheng (2010).
} 
that there are more trend followers than fundamentalists at the fundamental steady state.

Denote $\gamma_{f}=\mu n_{f}^{*} \beta_{f}$ and $\gamma_{c}=\mu\left(1-n_{f}^{*}\right) \beta_{c}$. The local stability and bifurcation of the fundamental steady state with respect to the time delay of system (3.1) are summarized in the following proposition].

Proposition 3.1. There exist $\tau_{0}$ and $\widetilde{\tau}$ with $0<\tau_{0}<\widetilde{\tau}$ such that the fundamental steady state $Q$ of system (3.1) is

(i) asymptotically stable for $\tau \in\left[0, \tau_{0}\right)$;

(ii) asymptotically stable for $\tau>\widetilde{\tau}$ when $\gamma_{f}+k>\gamma_{c}$;

(iii) unstable for $\tau>\widetilde{\tau}$ when $\gamma_{f}+k<\gamma_{c}$.

In addition, system (3.1) undergoes Hopf bifurcations at the zero solutions of functions $S_{n}^{ \pm}(\tau)$.

Note that the conditions in (ii) and (iii) of Proposition 3.1 are sufficient but not necessary. Specifically, the stability for the case $\tau_{0}<\tau<\widetilde{\tau}$ is completely determined by the functions $S_{n}^{ \pm}(\tau)$ and the system may switch between stability and instability for many times for $\tau \in\left(\tau_{0}, \widetilde{\tau}\right)$. We refer to Theorem 3.3 in He et al. (2009) for the properties of functions $S_{n}^{ \pm}(\tau)$. Proposition 3.1 implies that the fundamental steady state is stable for either small or large time delay when the market is dominated by the fundamentalists (in the sense of $\gamma_{f}+k>\gamma_{c}$ ). Otherwise, when the trend followers become more active comparing to the fundamentalists (in the sense of $\gamma_{c}>\gamma_{f}+k$ ), the fundamental steady state becomes unstable through Hopf bifurcations as the time delay increases. However, the effect of an increase in $k$, corrisponding to a larger weight of the most recent historical prices for trend followers, the fundamental price is stabilized. In the extreme case of $k \rightarrow \infty$, the price trend is given by current price. In this case, the demand of the trend followers (2.3) becomes zero and hence the system (3.1) becomes globally stable. This is consistent with the discrete-time HAMs, as the one by Chiarella, Dieci, He and Li (2013), which demonstrate that a decrease in the decay rate destabilizes the system. In line with the

\footnotetext{
${ }^{7}$ The definitions of parameters $\tau_{0}, \tilde{\tau}$, functions $S_{n}^{ \pm}(\tau)$ and the proof of Proposition 3.1 are given in Appendix B
} 
discrete-time HAMs, when the time horizon is small, the insignificant price trend, resulting in weak trading signals for the trend followers, limits the destabilizing activity of the chartists. Consequently, the fundamentalists dominate the market and the market price is stabilized to the fundamental value. However, Proposition 3.1 also indicates a very interesting phenomenon of the continuous-time model that is not easy to obtain in discrete-time models, which is the stability switching when the fundamentalists dominate the market 8 . That is, the system becomes unstable as the time delay increases initially, but the stability can be recovered when the time delay becomes large enough. Intuitively, when time horizon is small, the price trend becomes less significant, which limits the destabilizing effort of the trend followers. As the time horizon increases, the price trend becomes more sensitive to the changes in market price and consequently the trend followers become more active and destabilize the market. However, as the time horizon becomes very large, the price trend becomes smooth and less sensitive to price changes. Therefore the trend followers become less active and then, because of the dominance of the fundamentalists, the market becomes stable.

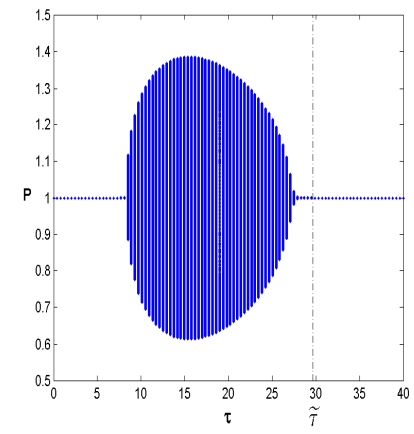

(a) Price bifurcation in $\tau$

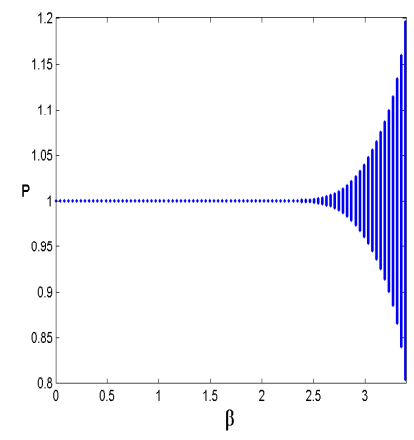

(b) Price bifurcation in $\beta$

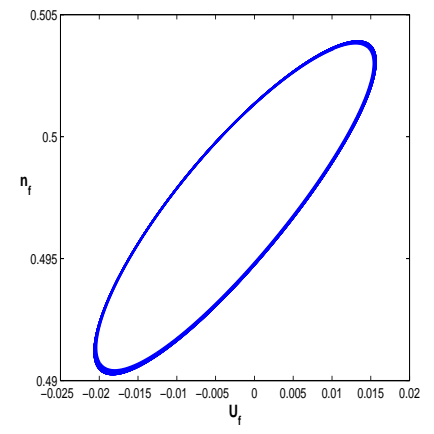

(c) Phase plot of $U_{f}$ and $n_{f}$

FiguRE 3.1. (a) The bifurcation of the market prices with respect to $\tau$ with $\beta=1$; (b) The bifurcation of market price with respect to $\beta$ with $\tau=8.3$; (c) The phase plot of the relationship between the fitness $U_{f}$ and the market fraction $n_{f}$ with $\tau=16$ and $\beta=1$.

\footnotetext{
${ }^{8}$ This phenomenon is also observed in the continuous-time model in He et al. (2009), He and Zheng (2010), and He and Li (2012).
} 
The simulation results of the nonlinear model (3.1) in Fig. 3.1 verify the stability results in Proposition 3.1] . Fig. 3.1 (a) plots the bifurcation diagram of the market price with respect to $\tau$, showing that the fundamental steady state is stable for $\tau \in$ $\left[0, \tau_{0}\right) \cup\left(\tau_{1}, \infty\right)$ and Hopf bifurcations occur at $\tau=\tau_{0} \approx 8.5$ and $\tau=\tau_{1} \approx 27$. This refers to the case that the market is dominated by fundamentalists. Fig. 3.1(b) plots the price bifurcation diagram with respect to the switching intensity parameter $\beta$. It shows that the steady state fundamental price is stable when the switching intensity $\beta$ is low, but becomes unstable as the switching intensity increases, bifurcating to stable periodic price with increasing fluctuations. This result shares the same spirit of the rational routes to complicated price dynamics in the discrete-time framework by Brock and Hommes (1997, 1998). Fig. 3.1 (c) illustrates the phase plot of $\left(U_{f}, n_{f}\right)$, showing the positive relation between the fitness $U_{f}$ and the market fraction $n_{f}$.

We also examine the impact of the different parameters on the stability. Proposition 3.1 shows that the decay rate $k$ plays a stabilizing role and simulations (not reported here) show that an increasing in $k$ narrows the instability interval by increasing the first bifurcation value and decreasing the second bifurcation value. Simulations also show that the system is always stable for all time horizons when $\mu$ is small but becomes unstable as $\mu$ increases. However, once the system becomes unstable, the stability seems insensitive to any further increase in $\mu$. The system can be stabilized by increasing $\beta_{f}$ and $C_{c}$, or decreasing $\beta_{c}, C_{f}$ and $\beta$. Proposition 3.1 shows that $\eta_{f}, \eta_{c}, \tau_{f}, \tau_{c}$ cannot affect the local stability and simulations show that their impact on the nonlinear system is very limited.

Fig. 3.2 provides further insights into the nonlinear dynamics of the market price and market fraction of the fundamentalists for $\tau=16$ when the fundamental steady state is unstable. Fig. 3.2 (a) illustrates the time series of the market prices $P(t)$ and the market fraction $n_{f}(t)$ of fundamentalists. It shows that the market fractions

\footnotetext{
${ }^{9}$ Unless specified otherwise, the following set of parameters are used in all the simulations in this paper: $k=0.05, \mu=1, \beta_{f}=1.4, \beta_{c}=1.4, \beta=1, C_{f}=0.05, C_{c}=0.03, \eta_{f}=0.5, \eta_{c}=0.6, \tau=$ $16, \tau_{f}=10, \tau_{c}=5, v=0.5$ and $\bar{F}=1$. Note that the fundamentalists generally focus on long-run performance but the trend followers focus on short-run performance. So we choose $\tau_{f}>\tau_{c}$ and correspondingly $\eta_{f}<\eta_{c}$.
} 


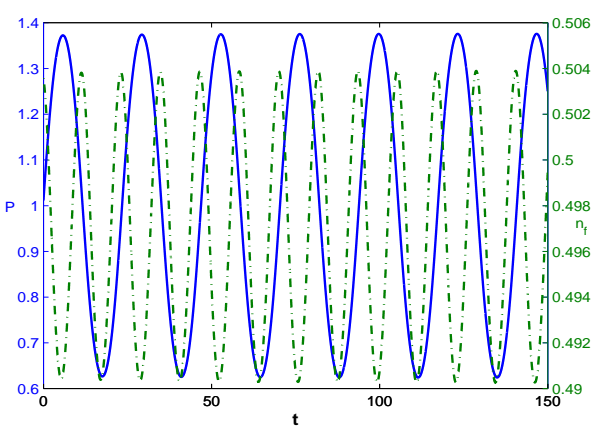

(a) Time series of $P$ and $n_{f}$

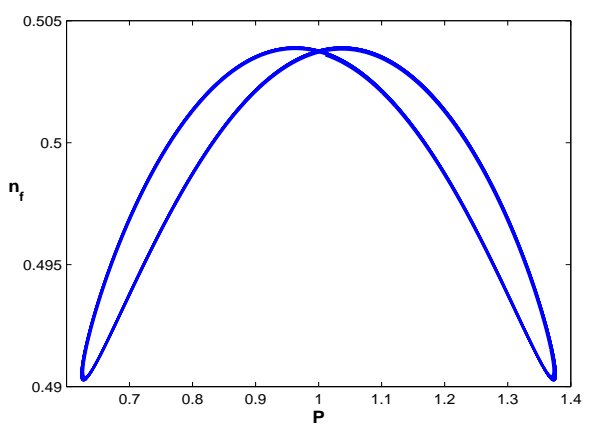

(b) Phase plot of $\left(P, n_{f}\right)$

Figure 3.2. (a) The time series of the market prices $P(t)$ (the blue solid line) and the market fraction $n_{f}(t)$ of fundamentalists (the green dash dot line) and (b) the phase plot of $\left(P(t), n_{f}(t)\right)$. Here $\tau=16$.

fluctuate with the market price. Fig. 3.2 (b) presents the phase plot of $\left(P(t), n_{f}(t)\right)$ showing that the price and fraction converge to a figure-eight shaped attractor, a phenomenon also observed in the discrete-time model by Chiarella et al (2006).

Interestingly, the herding parameter $v$ does not affect the local stability of the fundamental price and the deterministic price dynamics are very similar to the results in HL model without herding. For illustration, we compare this model with the HL model. In Appendix C, corresponding to Figs. 3.1 and 3.2, we present Figs. C.1 and C.2, respectively, for the HL model. It is observed that both models exhibit similar deterministic dynamics in price and market fraction. However, the nonlinear dynamics can be affected by the herding parameter $v$. Comparing Fig. 3.2 with $v=0.5$ and Fig. C.3 with $v=0.1$ in Appendix C, we observe that, when the herding behavior among agents is not very strong (as indicated by a decrease in the parameter $v$ ), the fluctuations of market price and, in particular, the market fractions of the fundamentalists are reduced. In other words, a strong herding among agents contributes to high fluctuations in market fractions, which then result in high volatility in market prices. This effect is further examined for the stochastic model in the next section. 


\section{Price Behavior of the Stochastic Model}

In this section, through numerical simulations, we examine the price dynamics of the stochastic model by focusing on the impact of three parameters: the time horizon $\tau$, the intensity of herding $v$, the switching intensity $\beta$ and the two noisy processes characterized by $\sigma_{F}$ and $\sigma_{M}$, on market volatility in both price and return. The analysis provides further insights into the different roles played by herding and switching in financial markets.

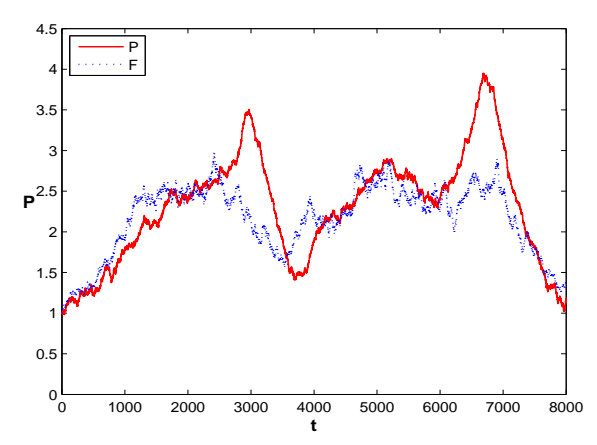

(a) Prices for $\tau=3$

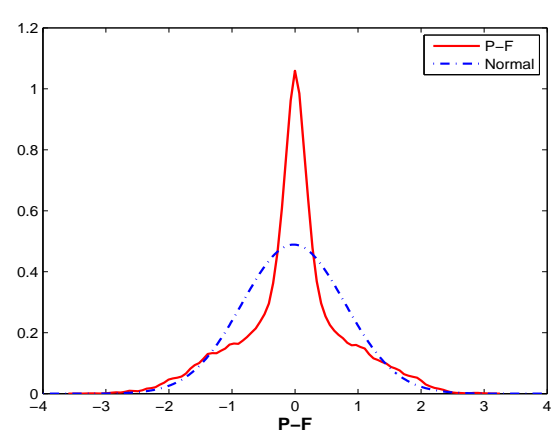

(c) Price deviation density for $\tau=3$

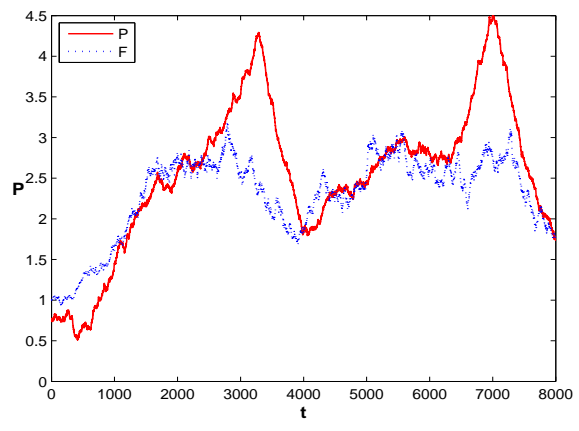

(b) Prices for $\tau=16$

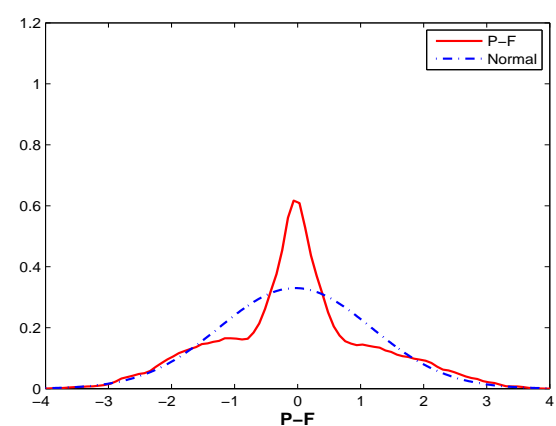

(d) Price deviation density for $\tau=16$

Figure 4.1. The time series of the fundamental price $F(t)$ (the blue dotted line) and the market prices $P(t)$ (the red solid line) with (a) $\tau=3$ and (b) $\tau=16$, and the distributions of the deviations of the market prices from the fundamental prices $P(t)-F(t)$ with (c) $\tau=3$ and (d) $\tau=16$. Here $\sigma_{F}=0.12$ and $\sigma_{M}=0.15$.

We first explore the interaction between the underlying deterministic dynamics and the two noisy processes by choosing two different values of the time horizon. For 
the deterministic model (3.1), Fig. 3.1 (a) shows that the time horizon can affect the stability of the fundamental price. In particular, the fundamental steady state is stable for $\tau=3$ and unstable for $\tau=16$, leading to periodic fluctuations of the market price. For the stochastic model, we choose the volatility of the fundamental price $\sigma_{F}=0.12$ and the volatility of the market noise $\sigma_{M}=0.1510$. With the same random draws of the fundamental price and market noise processes, we plot the fundamental price (the blue dotted line) and the market prices (the red solid line) in Fig. 4.1 for the two different values of $\tau$ under the same set of parameters for Fig. 3.1 (a). For $\tau=3$ and $\tau=16$, Figs. 4.1 (a) and (b) show that the market prices fluctuate around the fundamental prices and the fluctuations for $\tau=16$ are significantly larger than that for $\tau=3$. This observation is further supported by the distribution plots of the deviations of the market prices from the fundamental prices $P(t)-F(t)$ in Fig. 4.1 (c) for $\tau=3$ and in Fig. 4.1 (d) for $\tau=16$. With the standard deviations of 0.8158 for $\tau=3$ and 1.2091 for $\tau=16$, the deviations are more spread for $\tau=16$. This is partially underlined by the change in the stability of the underlying deterministic dynamics. Further simulations (not reported here) show that when the time horizon increases further to the stabilizing range indicated by Fig. 3.1 (a), the fluctuations of the market price deviations from the fundamental price become even more significant. This result illustrates that, when the underlying deterministic dynamics are stable, the stochastic dynamics can become very unstable with large fluctuations in price deviations due to a slow convergence of the market price to the fundamental price of the underlying deterministic model and its interaction with the fundamental and market noises. Therefore, an increase in time horizon increases the deviations of the market price from the fundamental price and the fluctuations of the market price.

To examine the effect of herding, with the same parameters and random draws, Fig. D.1 in Appendix D illustrates the corresponding results of the HL model without herding. It displays similar price patterns but with less significant deviations

\footnotetext{
${ }^{10}$ The constraint $n_{f}(t) \in[0,1]$ is imposed when simulating the stochastic system. In all the simulations, the time unit is one year and the time step corresponds to one day. Unless specified otherwise, an annual volatility of $\sigma_{F}=0.12$ and a market noise volatility of $\sigma_{M}=0.15$ are used in the paper.
} 
in prices (with the standard deviations of 0.2477 for $\tau=3$ and 0.4703 for $\tau=16$ ). The comparison implies that the herding behavior contributes to the excess volatility of the market price, a higher acceleration to market highs and lows, and a quicker mean reversion to the fundamental price.

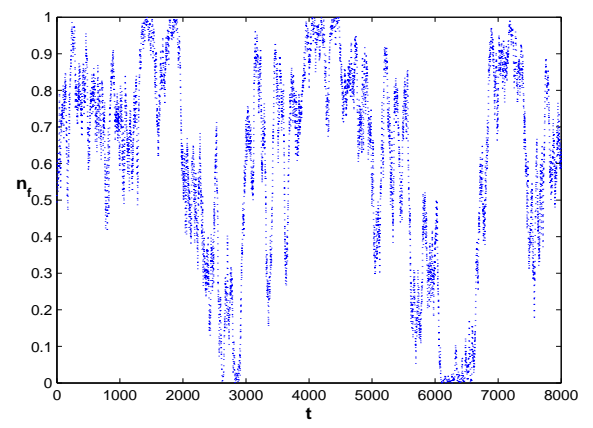

(a) Market fraction for $\tau=3$

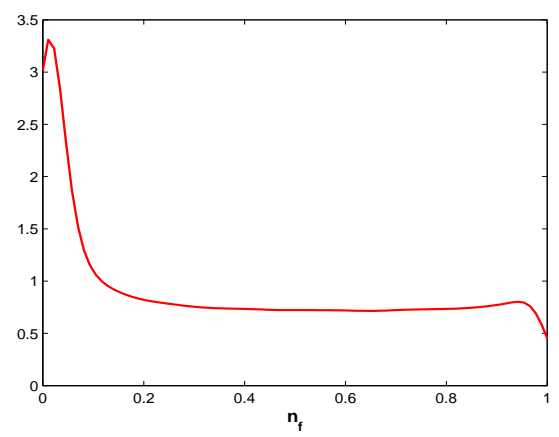

(c) Market fraction density for $\tau=3$

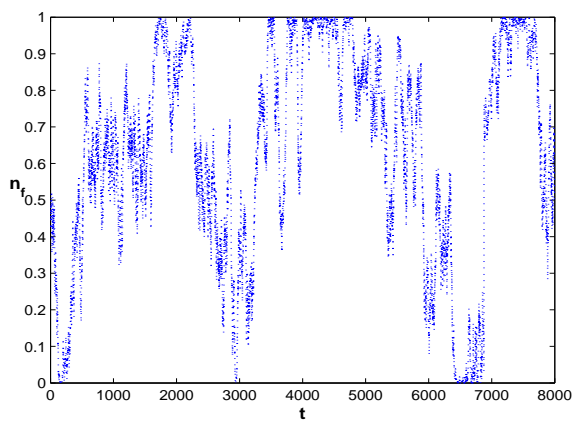

(b) Market fraction for $\tau=16$

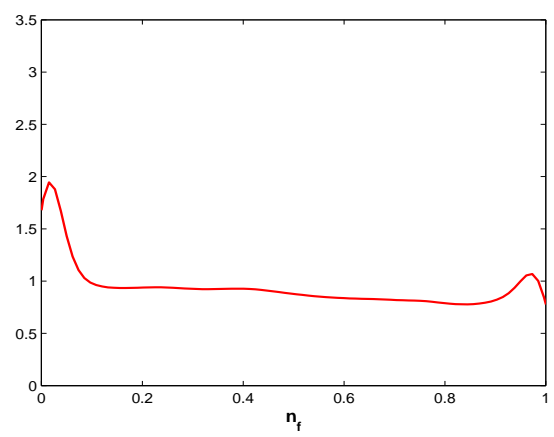

(d) Market fraction density for $\tau=16$

FiguRE 4.2. The time series of the fractions of the fundamentalists with (a) $\tau=3$ and (b) $\tau=16$ and the corresponding distributions with (c) $\tau=3$ and (d) $\tau=16$. Here $\sigma_{F}=0.12$ and $\sigma_{M}=0.15$.

We also plot the corresponding time series and distributions of the market fractions of the fundamentalists in Fig. 4.2 for both $\tau=3$ (the left panel) and $\tau=16$ (the right panel). Comparing to Fig. D.2 in Appendix D for the HL model (with switching but without herding), we have two observations. (i) In the presence of herding, the market fractions fluctuate wildly between 0 and 1 and are almost uniformly distributed except for the spike near 0, as shown in Fig. 4.2. However, without herding, the market fractions fluctuate around the steady state $\left(n_{f}=0.495\right)$ in a small range (from $35 \%$ to $75 \%$ ), illustrated by both the time series and distribution 
plots in Fig. D.2. This implies that the herding effect dominates the switching effect in generating high fluctuations in the market fractions. (ii) For the market fraction, by comparing the time series and distributions in Fig. 4.2 for $\tau=3$ (the left panel) and $\tau=16$ (the right panel), the effect of the time horizon is not highly significant. However, as indicated by the small peak near 1 for $\tau=16$ in Fig. 4.2 (d), the time horizon does affect the market fractions. In general we observe that herding leads to the dominance of the trend followers in the market, while an increase in the time horizon reduces the dominance of the trend followers and increases the dominance of the fundamentalists.

One of the innovative features of the model is that the market fraction is determined by the master equation (2.15) with endogenous volatility (2.16). Because of the dependence of the transition rates on the time horizon $\tau$, the switching $\beta$ and herding $v$, the volatility also depends on $\tau, \beta$ and $v$. Therefore the variations in the market fractions can affect the deviation of the market price from the fundamental price and return volatility. In general, the impact on market volatility can be different for price and return. In order to provide further insights into the different roles of herding, switching and time horizon on the fluctuations of the market fractions and the market volatility, we perform a separate sensitivity analysis for each of the three parameters $\tau, v$ and $\beta$ by keeping the others at their benchmark values. In each case, we examine the impact on the endogenously determined volatility of the market fractions (of the fundamentalists), $\sigma_{n_{f}}$, the volatility of the price deviations, $\sigma(P-F)$, and the volatility of the market returns, $\sigma(r)$. Based on the common set of the parameters, we run 100 simulations for each parameter combination and plot the averages of $\sigma_{n_{f}}, \sigma(P-F)$ and $\sigma(r)$ and denote by $\bar{\sigma}_{n_{f}}, \bar{\sigma}(P-F)$ and $\bar{\sigma}(r)$, respectively.

4.1. The effect of the time horizon. For $\tau \in[0,20]$, we conduct Monte Carlo simulations and plot $\bar{\sigma}_{n_{f}}, \bar{\sigma}(P-F)$ and $\bar{\sigma}(r)$ in the upper panel for $\beta=0.1,1,2$ and the lower panel for $v=0.01,0.1,0.5$ in Fig. 4.3, from which we can draw two observations about the volatility. 
(i) All the volatilities, in terms of $\bar{\sigma}_{n_{f}}, \bar{\sigma}(P-F)$ and $\bar{\sigma}(r)$, increase as the time horizon and herding increase 11 . For $\tau=0$, the trend followers are not participating in the market and the lower volatilities simply reflect the resulting volatilities of the market noise and fundamental noise. In this case, herding plays no role in market volatility in both the price and return (as indicated by the constant volatility for various $\beta$ and $v$ when $\tau=0$ in the middle and right panels). As $\tau$ increases, all the volatilities increase significantly. This effect becomes less significant for the market fraction volatility $\bar{\sigma}_{n_{f}}$ after an initial increase in $\tau$ (from 0 to about 5 ). The increase of the price deviation volatility in the time horizon is underlined by the destabilizing effect of $\tau$ on the underlying deterministic dynamics. Because of the fluctuations in the market fractions, we observe an increase in the return volatility as well. We also observe the same effect as the herding parameter $v$ increases in the low panel of Fig. 4.3. Due to the independence of the local stability from the intensity of herding, this result is more a consequence of the interaction of nonlinearity and noises.

(ii) Switching has a non-monotonic impact on the volatilities of market fraction and market price, but reduces the return volatility as the switching intensity increases. Figs. 4.3 (a) and (b) show that the volatilities of market fraction and market price deviations are non-monotonic as $\beta$ increases. An initial increase in $\beta$ leads to an increase in the volatility of market fraction but the effect reverses as $\beta$ increases further, implying a "hump" shaped effect on the volatilities of market fraction. However, we also observe an "U"-shaped effect on the volatility of the price deviations. This non-monotonic feature is explored further in the discussion below. Furthermore, Fig. 4.3 (c) shows that the volatilities of market return decrease in $\beta$, an opposite effect to herding. Such different impact of the switching and herding on volatility of price and return has not been explored in the literature. This provides some insights into the distinct role played by switching and herding in market volatility. If one argues that the switching behavior is more rational than the herding one, this result then indicates that herding can increase the return volatility, while switching can reduce the return volatility.

\footnotetext{
${ }^{11}$ Except $\bar{\sigma}_{n_{f}}$ for large $\beta$ that the volatility seems to decrease as time horizon increases. This is related to the non-monotonic impact of $\beta$ on the volatilities illustrated in Fig. 4.5.
} 


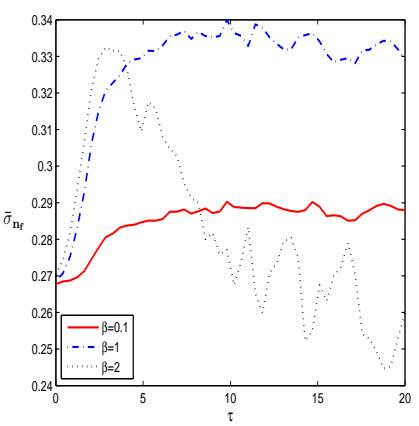

(a) $\bar{\sigma}_{n_{f}}$ in $\tau$ and $\beta$

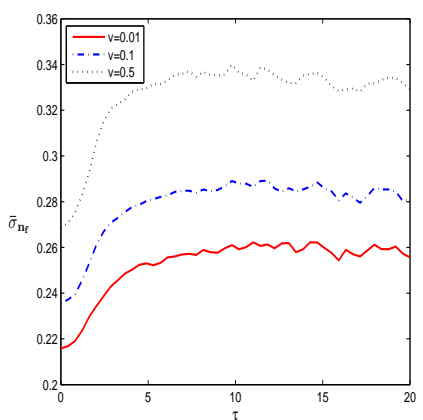

(d) $\bar{\sigma}_{n_{f}}$ in $\tau$ and $v$

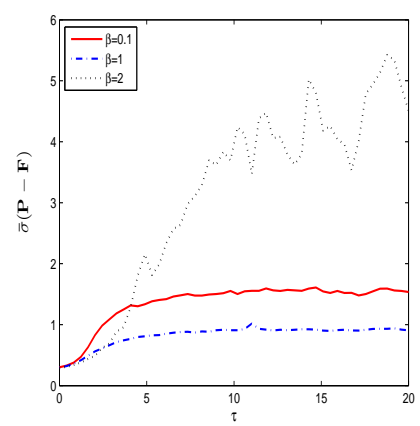

(b) $\bar{\sigma}(P-F)$ in $\tau$ and $\beta$

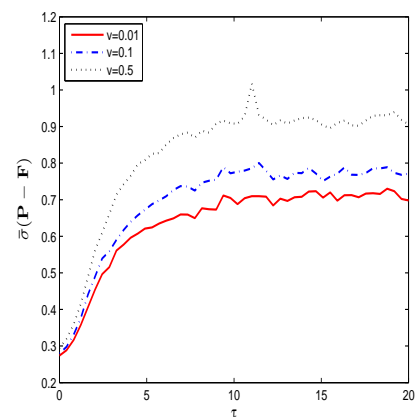

(e) $\bar{\sigma}(P-F)$ in $\tau$ and $v$

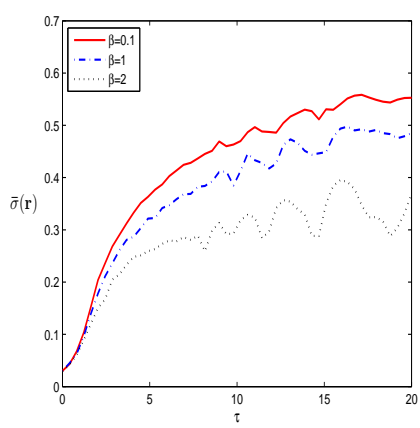

(c) $\bar{\sigma}(r)$ in $\tau$ and $\beta$

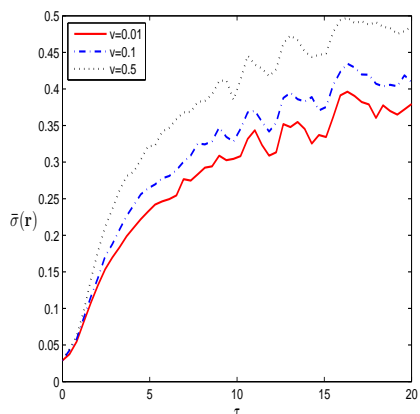

(f) $\bar{\sigma}(r)$ in $\tau$ and $v$

FigURE 4.3. The average variations of market fraction volatility $\bar{\sigma}_{n_{f}}$ (a) and (d), price deviation volatility $\bar{\sigma}(P-F)(\mathrm{b})$ and (e), and return volatility $\bar{\sigma}(r)(\mathrm{c})$ and (f) with $\beta=0.1,1,2$ (and $v=0.5$ ) in the upper panel and $v=0.01,0.1,0.5$ (and $\beta=1$ ) in the lower panel, respectively, with respect to time horizon $\tau \in[0,20]$.

4.2. The effect of the herding. For $v \in[0,1]$, we plot $\bar{\sigma}_{n_{f}}, \bar{\sigma}(P-F)$ and $\bar{\sigma}(r)$ for $\tau=0,3,16$ in the upper panel and for $\beta=0.1,1,2$ in the lower panel in Fig. 4.4 based on Monte Carlo simulations. It provides consistent observations as in the previous case. When the herding parameter $v$ and the time horizon $\tau$ increase, all the volatilities increase. However an increase in the switching parameter reduces the average volatility of return, as illustrated in Fig. 4.4 (f). Also Figs. 4.4 (d) and (e) show that the switching has a non-monotonic impact on the volatilities of market fraction and price. Consistently with the previous observations, the herding increases the fluctuations of the market price from the fundamental price and return. 


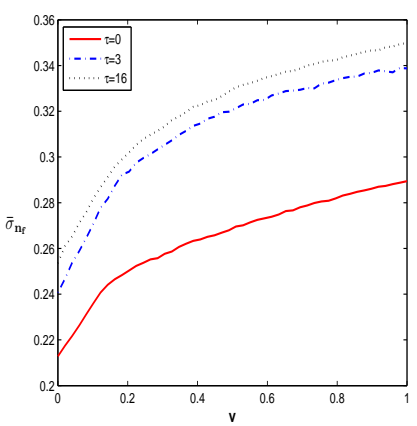

(a) $\bar{\sigma}_{n_{f}}$ in $v$ and $\tau$

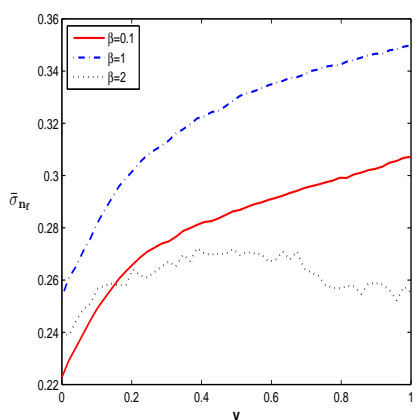

(d) $\bar{\sigma}_{n_{f}}$ in $v$ and $\beta$

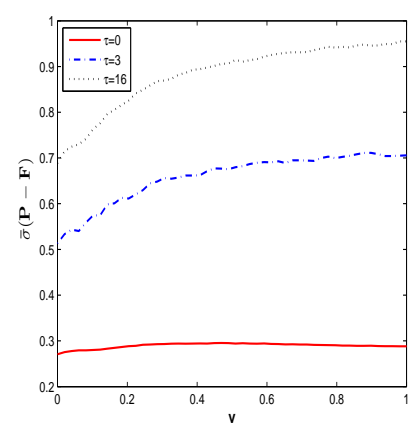

(b) $\bar{\sigma}(P-F)$ in $v$ and $\tau$

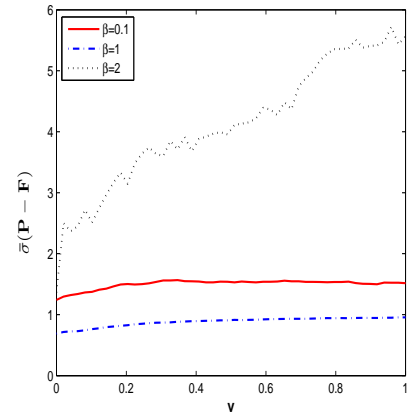

(e) $\bar{\sigma}(P-F)$ in $v$ and $\beta$

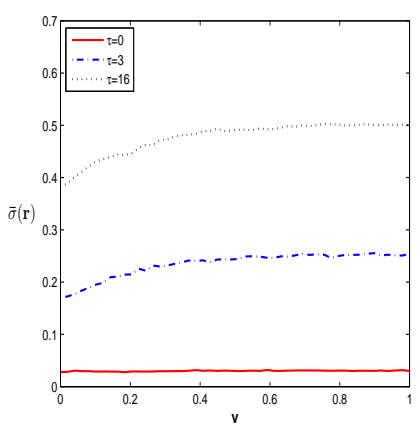

(c) $\bar{\sigma}(r)$ in $v$ and $\tau$

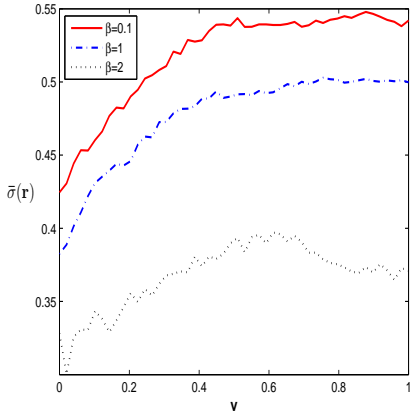

(f) $\bar{\sigma}(r)$ in $v$ and $\beta$

FigURE 4.4. The average variations of market fraction volatility $\bar{\sigma}_{n_{f}}$ (a) and (d), price deviation volatility $\bar{\sigma}(P-F)$ (b) and (e), and return volatility $\bar{\sigma}(r)(\mathrm{c})$ and (f) with $\tau=0,3,16$ (and $\beta=1$ ) in the upper panel and $\beta=0.1,1,2$ (and $\tau=16$ ) in the lower panel, respectively, with respect to $v \in[0,1]$.

4.3. The effect of switching. For $\beta \in[0,2]$, we plot $\bar{\sigma}_{n_{f}}, \bar{\sigma}(P-F)$ and $\bar{\sigma}(r)$ for $\tau=0,3,8,16$ in the upper panel and for $v=0.01,0.1,0.5$ in the lower panel in Fig. 4.5 based on Monte Carlo simulations. When $\beta=0$, the market fractions are driven purely by the herding behavior. In general, we observe consistent results in terms of the impact of time horizon and herding obtained in the previous two cases. However, there is a significantly non-monotonic relationship between the volatilities and the switching intensity $\beta$. We observe a "hump" shaped volatility in the market fraction (in Figs. 4.5 (a) and (d)), an "U"-shaped price volatility (in Figs. 4.5 (b) and (e)), and a decreasing volatility in returns (in Figs. 4.5 (c) and (f)) as the switching parameter $\beta$ increases. Interestingly, an initial increase in the switching leads to 


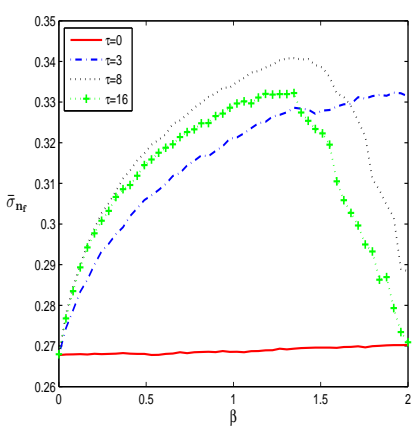

(a) $\bar{\sigma}_{n_{f}}$ in $\beta$ and $\tau$

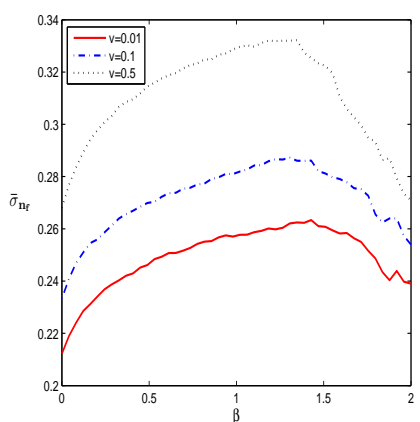

(d) $\bar{\sigma}_{n_{f}}$ in $\beta$ and $v$

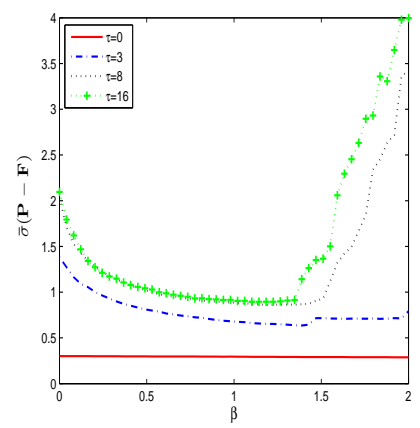

(b) $\bar{\sigma}(P-F)$ in $\beta$ and $\tau$

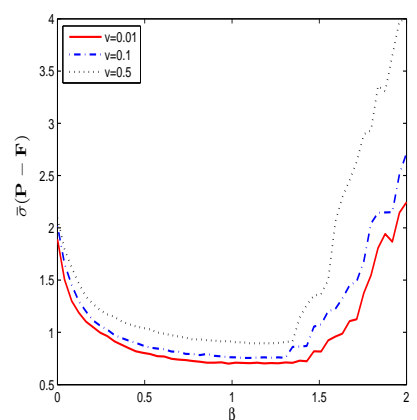

(e) $\bar{\sigma}(P-F)$ in $\beta$ and $v$

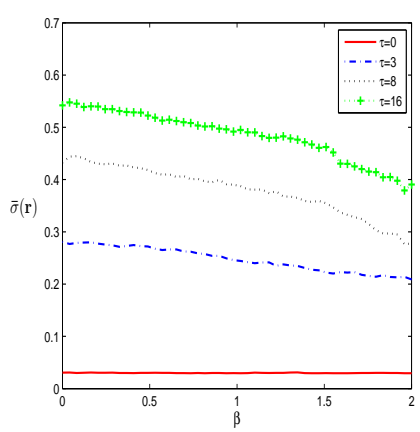

(c) $\bar{\sigma}(r)$ in $\beta$ and $\tau$

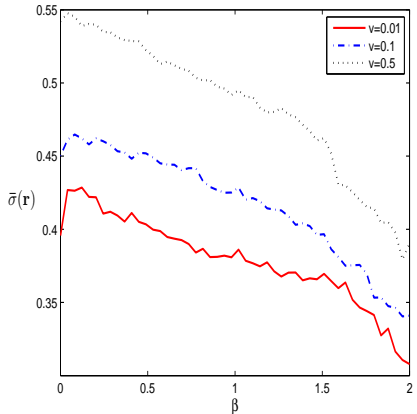

(f) $\bar{\sigma}(r)$ in $\beta$ and $v$

FIGURE 4.5. The average variations of market fraction volatility $\bar{\sigma}_{n_{f}}$ (a) and (d), price deviation volatility $\bar{\sigma}(P-F)(\mathrm{b})$ and (e), and return volatility $\bar{\sigma}(r)(\mathrm{c})$ and (f) with $\tau=0,3,16$ (and $v=0.5$ ) in the upper panel and $v=0.01,0.1,0.5$ (and $\tau=16$ ) in the lower panel, respectively, with respect to $\beta \in[0,1]$.

higher market fraction volatility and lower market price volatility, following by the decreasing volatility in fractions and the increasing volatility in price deviations when $\beta$ increases beyond certain threshold value. This result explains the phenomenon in Fig. 4.3 (a) that for large $\beta$, an increase in the time horizon $\tau$ leads to an initial increase in the market fraction volatility, but a dramatic decline when $\tau$ increases further. It also implies that large fluctuations in the market fractions reduce the market price deviation from the fundamental price when the switching intensity is low, but the effect becomes opposite when the switching intensity is high. However, a large intensity of switching always reduces return volatility. Therefore, we can 
have a market with high fluctuations in the market price and low volatility in the returns at the same time.

In summary, the impact on the volatility can be very different for price and returns. The trend chasing over a long time horizon and herding always lead to high volatility. However the switching and herding behaviors have an opposite effect on the market returns volatility. Although the switching has significant and nonmonotonic impact on the market volatility, it can actually reduce return volatility. This analysis explains the different effect of herding and switching mechanisms on the volatilities in price and returns.

\section{Power-law Behavior in Volatility}

After exploring the impact of the time horizon, herding, and switching on market volatility in the previous section, we are now interested in their impact on the powerlaw behavior in volatility. It has been well explored in the HAM literature that it is the interaction of the nonlinear dynamics of the underlying deterministic model and the noises that generate the power-law behavior. Both the switching and the herding mechanisms have been explored, but a comparison of different mechanism is missing in the literature. This section is devoted to such a comparison. We first examine the impact of the noises and then of the time horizon, switching and herding on the ACs of the returns, absolute returns, and squared returns with the two noises.

To motivate the analysis, we first present the ACs of the returns, absolute returns, and squared returns for market daily closing price indices of the DAX 30, the FTSE 100, the NIKKEI 225, and the S\&P 500 from 01/02/1984 to 31/07/2013 from Datastream in Fig. 5.1. Note that the levels of all the ACs for the returns are not significant, but they are significant and decaying for the absolute and squared returns. This phenomenon is referred as the power-law behavior or long memory in market volatility in empirical literature, see He and Li (2007) and references cited there. 


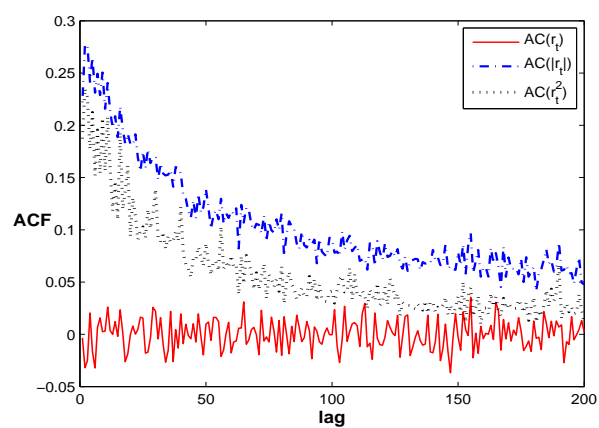

(a) DAX 30

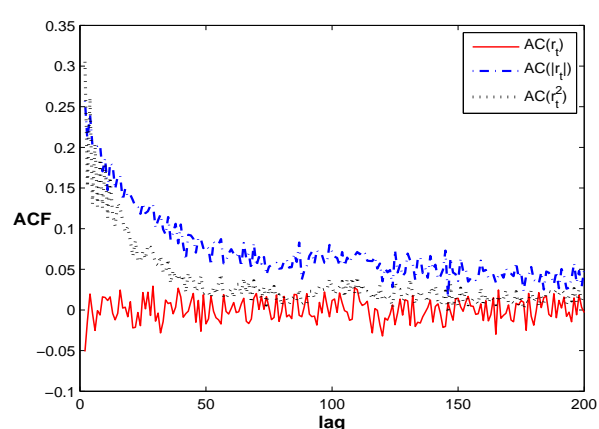

(c) NIKKEI 225

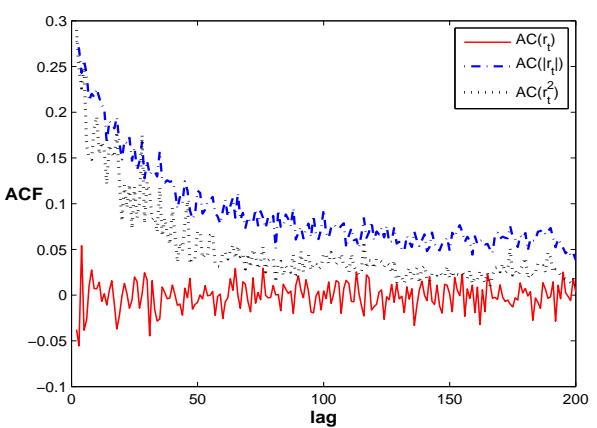

(b) FTSE 100

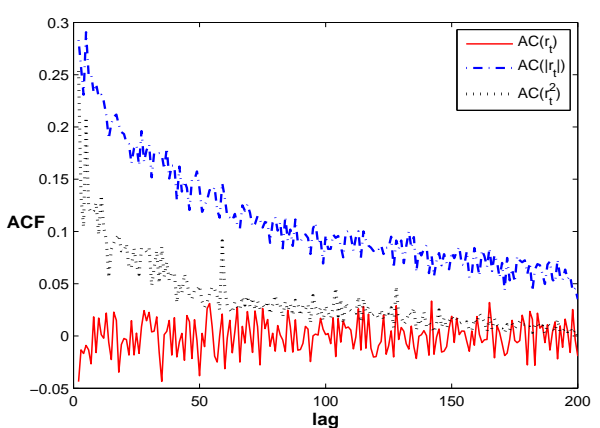

(d) S\&P 500

Figure 5.1. The ACs of the returns (the bottom lines), the squared returns (the middle lines) and the absolute returns (the upper lines) for (a) the DAX 30, (b) the FTSE 100, (c) the NIKKEI 225, and (d) the S\&P 500.

5.1. The effect of the noises. For the two exogenously given fundamental and market noises, we examine the impact of the noises by considering three combinations of (i) both the fundamental and market noises; (ii) the market noise only; and (iii) the fundamental noise only.

We first consider the effect of the fundamental and market noises with $\sigma_{F}=0.12$ and $\sigma_{M}=0.15$. Fig. E.1 in Appendix E represents the results of a typical simulation based on the same set of parameters in Fig. 4.1 with $\tau=16$. The results demonstrate that the stochastic model established in this paper is able to generate market price deviations from the fundamental value (in Fig. E.1 (a)), most of the stylized facts observed in financial markets (including volatility clustering in Fig. E.1 (b), high kurtosis in in Fig. E.1 (c)), and the power-law behavior in volatility (insignificant 
autocorrelation (AC) levels for returns in Fig. E.1(d), but significant decaying AC levels for the absolute returns and the squared returns in Figs. E.1 (e) and (f)).

Next we consider the effect of the market noise. With the same parameters and random seeds, Fig. E.2 in Appendix E shows that the model is able to generate a similar result to the previous case with the two noises, although the levels of the significant ACs are lower. This implies that, even with a constant fundamental value, the model has a great potential in generating the power-law behavior. This result is significantly different from the switching HL model, in which the model is not able to generate the power-law behavior without fundamental noise.

Finally, we consider the effect of the fundamental noise. Fig. E.3 in Appendix E shows that the model is not able to generate the volatility clustering and the power-law behavior, which is consistent with the HL model. Meanwhile the market returns, absolute returns and squared returns exhibit highly significant ACs with strong decaying patterns, which is mainly due to the strong effect of the deterministic dynamics of the price process.

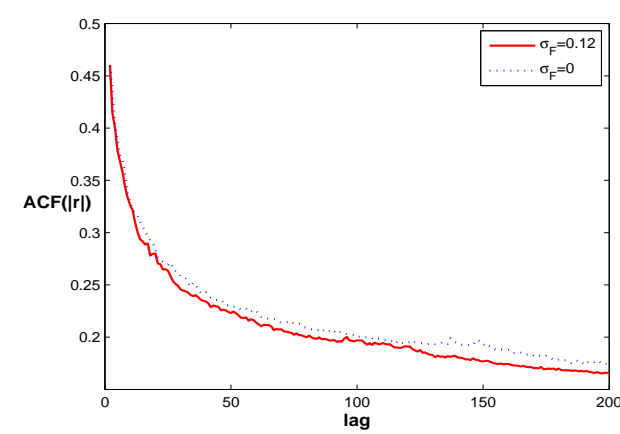

(a) The ACs of the absolute returns

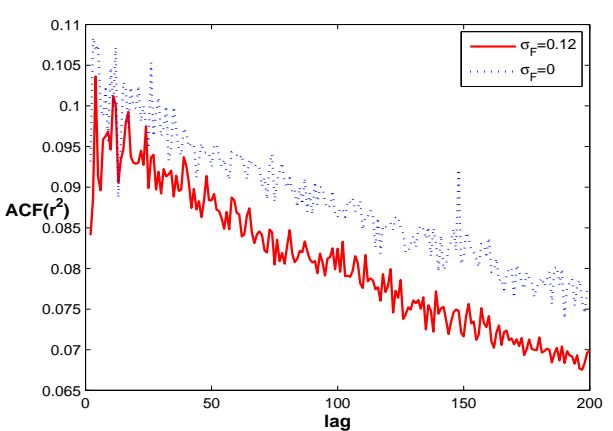

(b) The ACs of the squared returns

Figure 5.2. The effect of the fundamental noise: the ACs of (a) the absolute returns and (b) the squared returns based on 100 simulations with $\tau=16, v=0.5$ for $\sigma_{F}=0.12$ (the red solid line) and $\sigma_{F}=0$ (the blue dash-dotted line).

To further investigate the effect of the fundamental noise on the AC patterns, Fig. 5.2 compares the ACs of the absolute returns and the squared returns for $\sigma_{F}=0.12$ (the red solid line) and $\sigma_{F}=0$ (the blue dash-dotted line) based on 100 simulations. 
The ACs of the absolute returns and squared returns are significant and decaying. However, with the market noise $\sigma_{F}=0.12$, the ACs decay quickly than those for $\sigma_{F}=0$. This indicates that the market noise plays a key role in generating the power-law behavior, though it is not the only factor, as argued in He and Li (2007).

The previous analysis on the effect of the time horizon, switching, and herding shows that they play different roles in generating volatility in market price and return. We now further investigate their effect on the power-law behavior. Similarly, we consider three cases, each focuses on one of the three parameters $\tau, v$ and $\beta$. For each case, we examine the impact on the AC patterns of the absolute and squared returns. Based on the common set of the parameters, we run 100 simulations for each parameter combination and plot the average ACs for the absolute and squared returns 12

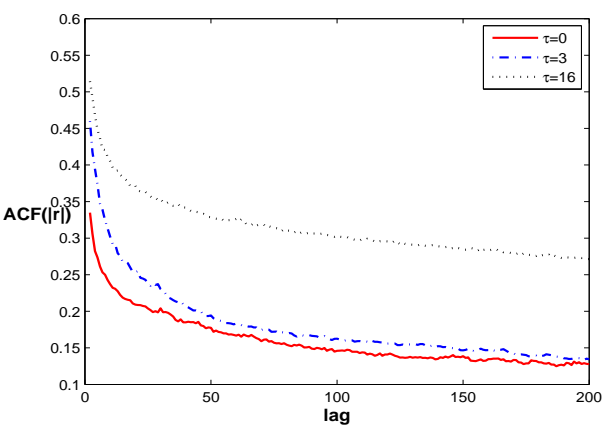

(a) The ACs of the absolute returns

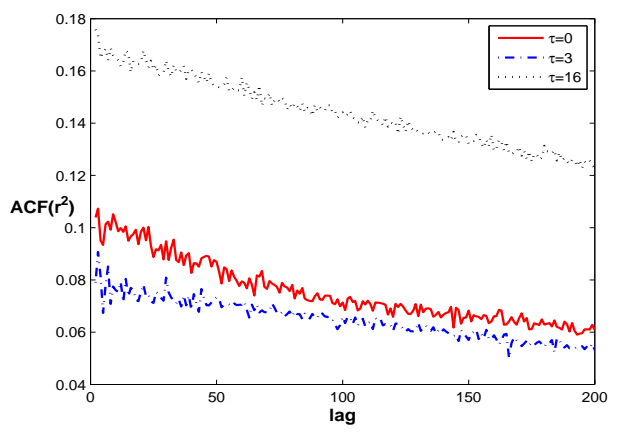

(b) The ACs of the squared returns

Figure 5.3. The effect of the time horizon: the ACs of (a) the absolute returns and (b) the squared returns based on 100 simulations with $v=0.5, \beta=1, \sigma_{F}=0.12, \sigma_{M}=0.15$ for $\tau=0, \tau=3$ and $\tau=16$.

5.2. The effect of the time horizon. First, we present in Fig. 5.3 the effect of the time horizon on the $\mathrm{AC}$ patterns for the absolute returns (the left panel) and the squared returns (the right panel). We observe that the trend chasing based on different time horizons contributes to the significant decaying AC patters for both the absolute and squared returns. Also the significant levels of the ACs increase

\footnotetext{
${ }^{12}$ The average AC levels for the returns are insignificant in all three cases reported.
} 
as the time horizon increases, in particular, when the time horizon is large. This suggests that a commonly observed slow decaying AC patterns in the discrete-time HAM literature (see for example He and Li (2007)) might be due to the long time horizons used for modeling the trend chasing. In other words, trend chasing based on short time horizons contributes to more realistic power-law behavior in volatility. Intuitively, technical analysis such as trend following strategy is mainly used for short-term investment comparing to the fundamental analysis for long-term investment. Therefore the trend chasing based on short-time horizon contributes to volatility in financial markets.

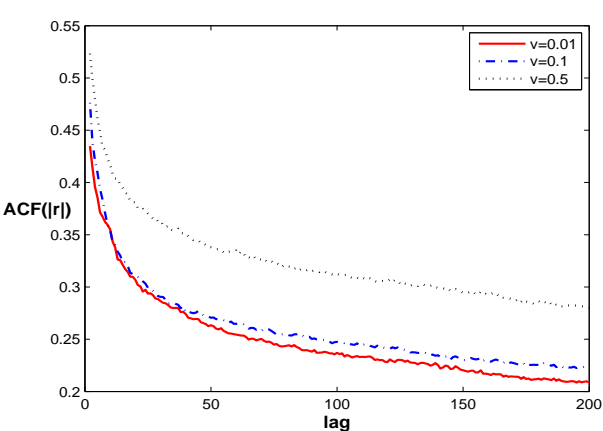

(a) The ACs of the absolute returns

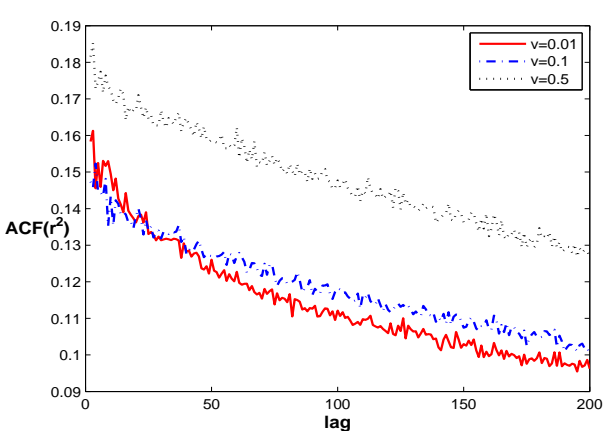

(b) The ACs of the squared returns

Figure 5.4. The effect of the herding: the ACs of (a) the absolute returns and (b) the squared returns based on 100 simulations with $\tau=16, \beta=1, \sigma_{F}=0.12, \sigma_{M}=0.15$ for $v=0.01, v=0.1$ and $v=0.5$.

5.3. The effect of the herding intensity. Secondly, we present in Fig. 5.4 the effect of the herding on the AC patterns for the absolute returns (the left panel) and the squared returns (the right panel), showing the contribution of the herding to the power-law behavior in volatility. Similar to the effect of the time horizon, an increase in the herding increases the level of the significant ACs for both the absolute and squared returns. However, differently from the effect of the time horizon, the ACs decay quickly under the herding.

5.4. The effect of the switching intensity. Thirdly, we present in Fig. 5.5 the effect of the switching on the AC patterns for the absolute and squared returns. It 


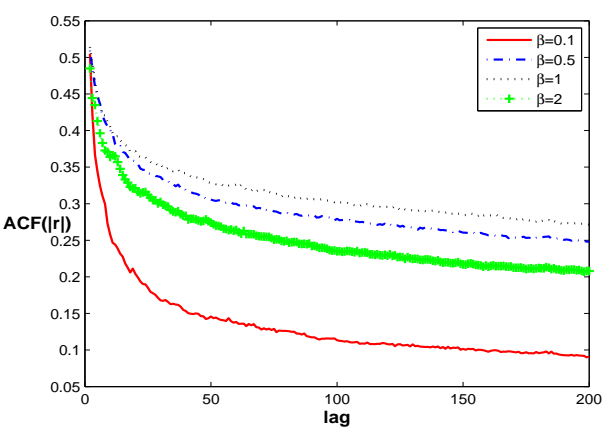

(a) The ACs of the absolute returns

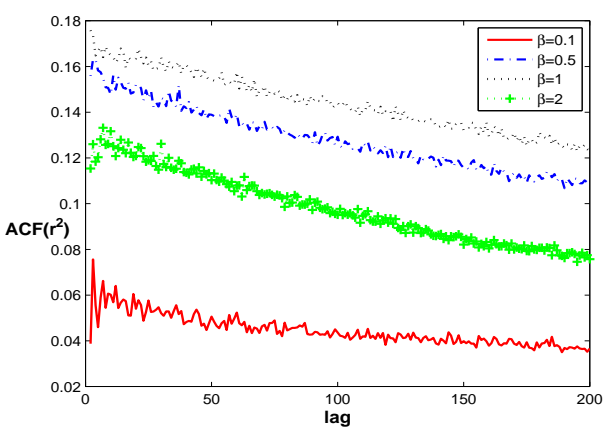

(b) The ACs of the squared returns

Figure 5.5. The effect of the switching: the ACs of (a) the absolute returns and (b) the squared returns based on 100 simulations with $v=0.5, \tau=16, \sigma_{F}=0.12, \sigma_{M}=0.15$ for $\beta=0.1, \beta=0.5, \beta=1$ and $\beta=2$.

shows that the switching contributes to the power-law behavior. Interestingly, and differently from the effect of the time horizon and herding, the level of the significant ACs for both the absolute and squared returns is not monotonic with respect to the switching intensity $\beta$. The level increases significantly when $\beta$ increases from 0.1 to 0.5 , and then less significantly when $\beta$ increases to 1 , but decreases when $\beta$ increases further to 2. In particular, the ACs for the absolute returns decay very quickly, comparing to the effect of the herding. This observation, together with the discussion in Section 4.3, suggests that an increase in the switching can reduce the return volatility and generate the power-law behavior at the same time. This provides further support on the explanatory power of the adaptive switching in financial markets initiated in Brock and Hommes (1998).

In summary, we have explored different mechanisms for the switching and the herding behavior of agents on the market volatility and power-law behavior in particular. We show that both contribute to the power-law behavior, however the effect is monotonically increasing with the herding, but not monotonic for the switching.

Finally, we investigate the question if pure herding is sufficient to explain the power-law behavior by considering the case with $\sigma_{F}=0$ and $\tau=0$. In this case, the fundamental value becomes constant and the trend followers become naive traders who take the current price as the expected future market price and hence do not 


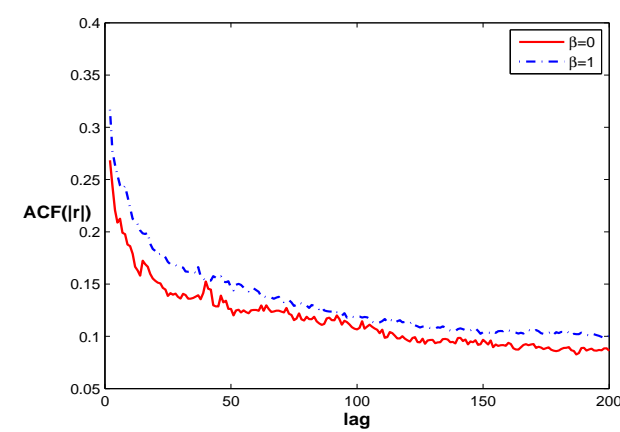

(a) The ACs of the absolute returns

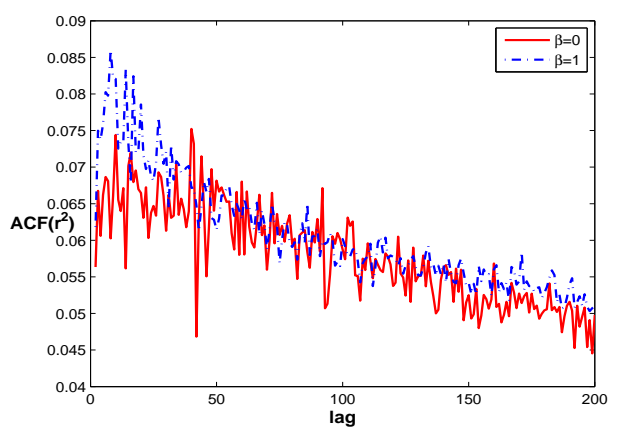

(b) The ACs of the squared returns

FigurE 5.6. The ACs of (a) the absolute returns and (b) the squared returns based on 100 simulations with $v=0.5, \tau=0, \sigma_{F}=0, \sigma_{M}=$ 0.15 for $\beta=0$ and $\beta=1$.

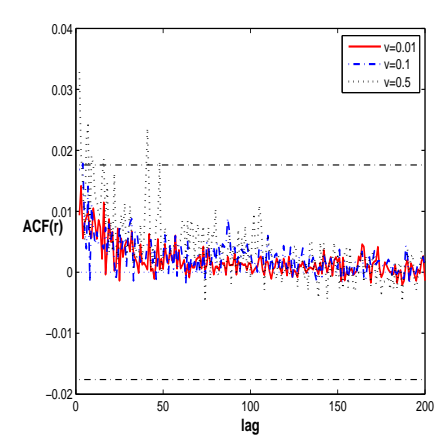

(a) $A C\left(r_{t}\right)$

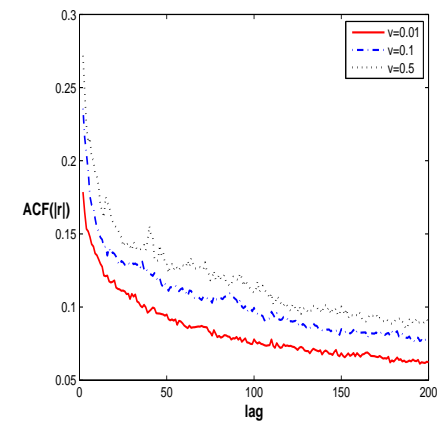

(b) $A C\left(\left|r_{t}\right|\right)$

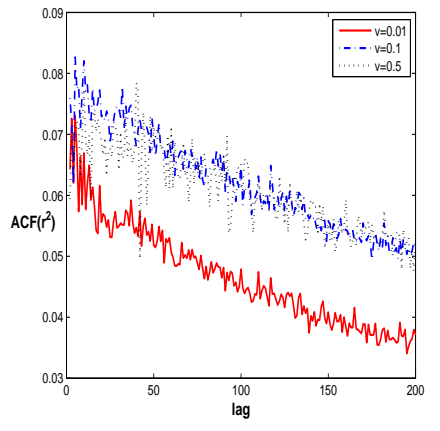

(c) $A C\left(r_{t}^{2}\right)$

Figure 5.7. The ACs of (a) the market returns; (b) the absolute returns and (c) the squared returns based on 100 simulations with $\tau=0, \beta=0, \sigma_{F}=0, \sigma_{M}=0.15$ for $v=0.01, v=0.1$ and $v=0.5$.

trade in the market anymore. Thus the market is driven by a pure herding mechanism. Fig. 5.6 illustrates the significant and decaying AC patterns in both the absolute and squared returns, although the AC level for the squared returns is significantly lower comparing to the cases discussed previously. Interestingly, there is no significant difference in the AC patters of the absolute return between no switching $(\beta=0)$ and the switching $(\beta=1)$. This result is consistent with Alfarano et al. (2005) who show that a pure herding model with fundamentalists and noise traders can generate the power-law behavior. As a robustness check, we present Fig. 5.7 
with three different values of $v$. Similar to Fig. 5.4, we observe more significant AC patterns as the herding parameter $v$ increases.

Comparing to the AC patterns of the market indices in Fig. 5.1, we may argue that the switching generates similar AC patterns to the NIKKEI 225 and the S\&P 500 with quickly decaying AC patterns, while the herding generates similar AC patterns to the DAX 30 and FTSE 300 with relatively slow decaying AC patterns. This observation may suggest different market behaviors in different markets and we leave this challenging empirical question to further stages of our research.

\section{Conclusion}

Market volatility is one of the most important features in financial markets and the question is what drives it. To answer this question, the most obvious way is to consider how agents behave in financial markets. Among various agents' behaviors in financial markets, trend chasing, adaptive switching and herding are the most important, well documented and studied in the empirical literature. This paper incorporates these three behavioral elements into an asset pricing model in a continuous-time framework and shows that they all contribute to market volatility in different manners.

Most of the asset pricing models with heterogeneous agents are in discrete-time framework focusing on trend chasing over short time horizon and adaptive switching. Herding is commonly modelled by the master equation in a continuous-time setting. Therefore the roles of trend chasing, switching and herding in market volatility have been studied in separate frameworks. Within a continuous-time framework, this is the first paper, to our knowledge, to combine trend chasing based on different time horizon, switching and herding together to examine their roles on market volatility in price and return. We show that both the herding and the trend chasing based on long time horizon increase the fluctuations of the market price deviation from the fundamental price and volatility of the market return. With respect to the switching, they reduce the volatility in returns but leads to a "U"-shaped price volatility as the switching intensity increases. Therefore herding and switching have an opposite effect on return volatility. 
We also examine the explanatory power of the model in generating the power-law behavior in return volatility. We show that, although the trend chasing, switching and herding all contribute to the power-law behavior, the significant levels for the ACs increase in the time horizon and herding, but an initial increase and then decrease when the switching intensity increases. In addition, with herding, the market noise plays an essential role in generating the power-law behavior.

The model proposed in this paper provides a unified framework to deal with trend chasing, switching and herding in financial markets. The results provide some further insights into possible different mechanisms for generating bubbles and crashes, excess volatility and power-law behavior in volatility. Whether a particular market is dominated by herding or switching is an empirical question that is left for future research. 


\section{Appendix A. Analytical solution for the master equation}

We solve the master equation using the approximation method introduced by Aoki (2002). Assume the fraction of fundamentalists in a given moment is determined by its expected mean $(m)$, the drift, and, an additive fluctuation component $s$ of order $1 / N^{1 / 2}$ around this value. Thus we can write

$$
\frac{N^{f}}{N}=m+\frac{1}{\sqrt{N}} s
$$

where $s$ is a standard white noise. The asymptotically approximate solution of the master equation is given by the system of coupled differential equations

$$
\begin{aligned}
\frac{d m}{d t}=\zeta(t) m & -[\zeta(t)+\xi(t)] m^{2} \\
\frac{\partial Q}{\partial t}=[2(\zeta(t) & +\xi(t)) m-\zeta(t)] \frac{\partial}{\partial s}(s Q(s, t)) \\
& +\frac{m[\zeta(t)+m(\xi(t)-1)]}{2} \frac{\partial^{2}}{\partial s^{2}} Q(s, t),
\end{aligned}
$$

where $Q(s, t)$ is the transition density function of the spread $s$ at time $t$. The first equation of (A.2) is a deterministic ordinary differential equation which displays logistic dynamics for the trend. The second equation is a second order stochastic partial differential equation, known as the Fokker-Planck equation that drives the spread component (i.e. the fluctuations around the trend) of the probability flow. By letting $m$ equal to its steady state $m^{*}=\frac{\zeta}{\zeta+\xi}$, we have the distribution function $\theta$ for the spread $s$, which is given by

$$
\theta(s)=C e^{-\frac{s^{2}}{2 \sigma^{2}}} \quad \text { with } \quad \sigma^{2}=\frac{\zeta \xi}{(\zeta+\xi)^{2}}
$$

which is a Gaussian density. Therefore, the two components of the dynamics of the proportion of fundamentalists as represented by (A.1) are quantified. Accordingly the evolution of the proportion of fundamentalists is given by the trend, described by (A.2), plus a stochastic noise distributed according to (A.3). So we have (2.15). For more details, we refer to Chiarella and Di Guilmi (2011a). 


\section{Appendix B. Definitions of the Parameters and Functions Used in Proposition 3.1}

The characteristic equation of the system (3.1) at the fundamental steady state is given by $13 \Delta(\lambda):=\left(\lambda+2 v n_{f}^{*}\right) \widetilde{\Delta}(\lambda)=0$, where

$$
\widetilde{\Delta}(\lambda)=\left(\lambda+\eta_{f}\right)\left(\lambda+\eta_{c}\right)\left[\lambda^{2}+\left(k+\gamma_{f}-\gamma_{c}\right) \lambda+k \gamma_{f}-k \gamma_{c}+\frac{k \gamma_{c}}{1-e^{-k \tau}}-\frac{k \gamma_{c} e^{-(\lambda+k) \tau}}{1-e^{-k \tau}}\right] .
$$

Note that equation (B.1) has the same form as the characteristic equation of the model studied in He and Li (2012). Hence we can apply Proposition 3.1 in He and Li (2012) to system (3.1). It follows that the stability of the steady state does not change for time delay $\tau>\tilde{\tau}$ with

$$
\widetilde{\tau}=\frac{1}{k} \ln \left[1+\frac{2 k \gamma_{c}}{\left(k+\gamma_{f}-\gamma_{c}\right)^{2}+2\left|k+\gamma_{f}-\gamma_{c}\right| \sqrt{k \gamma_{f}}}\right] .
$$

That is, there is an upper bound on the time delay for stability change. The change in stability happens only for $\tau \in[0, \widetilde{\tau}]$ if there exists a non-negative integer $n$ such that $S_{n}^{+}(\tau)=0$ or $S_{n}^{-}(\tau)=0$. Here 14

$$
S_{n}^{ \pm}(\tau)=\tau-\frac{\theta_{ \pm}(\tau)+2 n \pi}{\omega_{ \pm}(\tau)}, \quad \tau \in(0, \widetilde{\tau}], \quad n=0,1,2, \cdots
$$

$\omega_{ \pm}=\left(\frac{-a_{1} \pm \sqrt{a_{1}^{2}-4 a_{2}}}{2}\right)^{\frac{1}{2}}, \quad \theta_{ \pm}(\tau)= \begin{cases}\arccos \left(a_{4 \pm}\right), & \text { for } a_{3 \pm} \geq 0 ; \\ 2 \pi+\arcsin \left(a_{3 \pm}\right), & \text { for } a_{3 \pm}<0, a_{4 \pm} \geq 0 \\ 2 \pi-\arccos \left(a_{4 \pm}\right), & \text { for } a_{3 \pm}<0,\end{cases}$

and

$$
\begin{aligned}
a_{1}=k^{2}+\gamma_{f}^{2}+\gamma_{c}^{2}-2 \gamma_{f} \gamma_{c}-\frac{2 k \gamma_{c}}{1-e^{-k \tau}}, & a_{2}=k^{2} \gamma_{f}^{2}+\frac{2 k^{2} \gamma_{f} \gamma_{c} e^{-k \tau}}{1-e^{-k \tau}} \\
a_{3 \pm}=\frac{-\omega_{ \pm}(\tau)\left(1-e^{-k \tau}\right)\left(k+\gamma_{f}-\gamma_{c}\right)}{k \gamma_{c} e^{-k \tau}}, & a_{4 \pm}=1-\frac{\left(1-e^{-k \tau}\right)\left(\omega_{ \pm}^{2}(\tau)-k \gamma_{f}\right)}{k \gamma_{c} e^{-k \tau}}
\end{aligned}
$$

Denote

$$
\tau_{0}=\inf \left\{\{\widetilde{\tau}\} \bigcup\left\{\tau \in(0, \widetilde{\tau}] \mid \exists n \in\{0,1,2, \cdots\}, S_{n}^{+}(\tau)=0 \text { or } S_{n}^{-}(\tau)=0\right\}\right\} .
$$

\footnotetext{
${ }^{13}$ Interestingly, the time delays $\tau_{f}, \tau_{c}$ introduced in the performance measures in (2.9) do not appear in the characteristic equation, hence they do not affect the local stability and bifurcation analysis. This is due to the fact that they are in higher order terms and they affect the nonlinear dynamics, rather than the dynamics of the linearized system.
}

${ }^{14}$ We refer to Theorem 3.3 in He et al. (2009) for the properties of functions $S_{n}^{ \pm}(\tau)$. 
Appendix C. Comparison to the HL model and Nonlinear Effect of HERDING

To examine the effect of herding, we present the corresponding results of noherding model in He and Li (2012), the HL model. Unless specified otherwise, we choose the parameter values $k=0.05, \mu=1, \beta_{f}=1.4, \beta_{c}=1.4, C_{f}=0.05$, $C_{c}=0.03, \eta_{f}=0.5, \eta_{c}=0.6, \tau_{f}=10, \tau_{c}=5$, and $\bar{F}=1$.

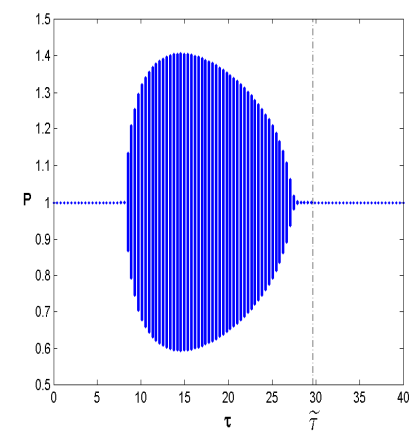

(a) Price bifurcation in $\tau$

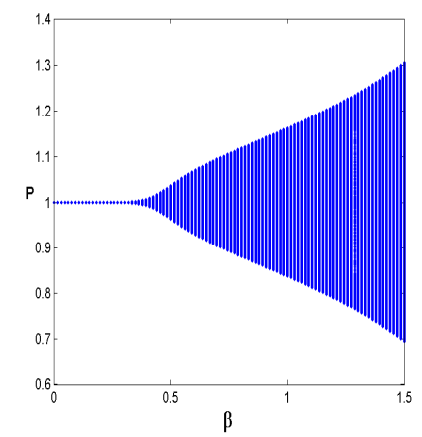

(b) Price bifurcation in $\beta$

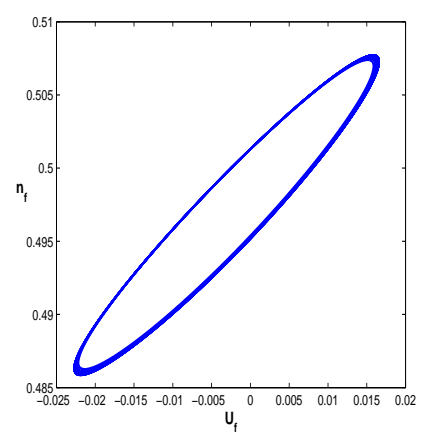

(c) Phase plot of $U_{f}$ and $n_{f}$

Figure C.1. (a) The bifurcation of the market prices with respect to $\tau$ with $\beta=1$; (b) The bifurcation of market price with respect to $\beta$ with $\tau=8$; (c) The phase plot of the relationship between the fitness $U_{f}$ and the market fraction $n_{f}$ with $\tau=16$ and $\beta=1$ for HL model.

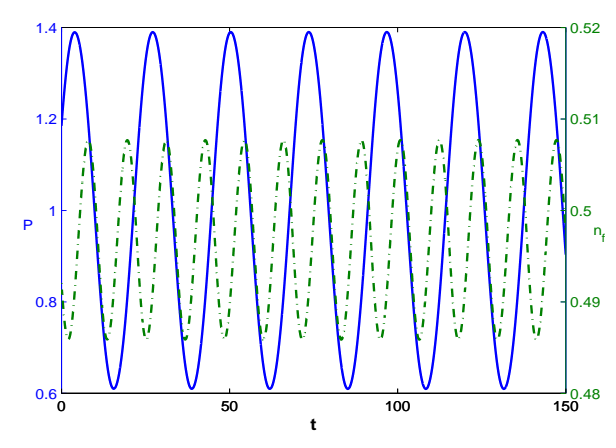

(a) Time series of $P$ and $n_{f}$

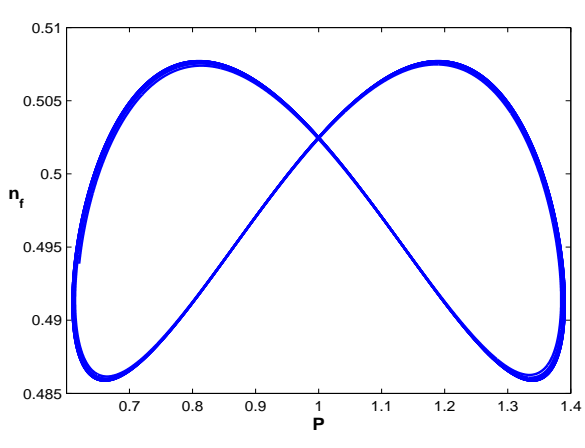

(b) Phase plot of $\left(P, n_{f}\right)$

Figure C.2. (a) The time series of the market prices $P(t)$ (the blue solid line) and the market fraction $n_{f}(t)$ of fundamentalists (the green dash dot line) and (b) the phase plot of $\left(P(t), n_{f}(t)\right)$. 


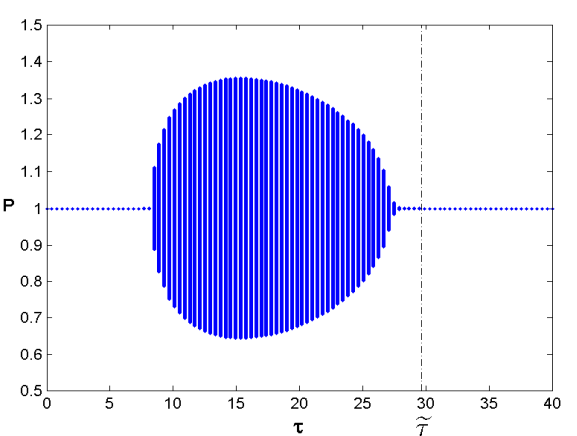

(a) Price bifurcation

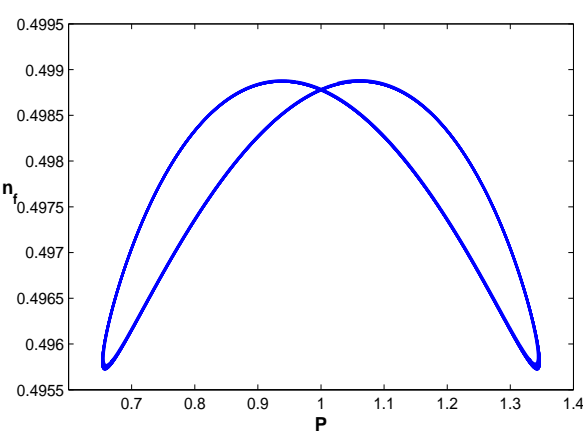

(b) Phase plot of $\left(P, n_{f}\right)$

Figure C.3. (a) The bifurcation diagram of the market prices with respect to $\tau$ for model (3.1) and (b) the corresponding phase plot of $\left(P(t), n_{f}(t)\right)$. Here $v=0.1$ and $\tau=16$. 


\section{Appendix D. Price Volatility Comparison to the HL Model}

This appendix presents some results from the HL model and price volatility of the herding model.

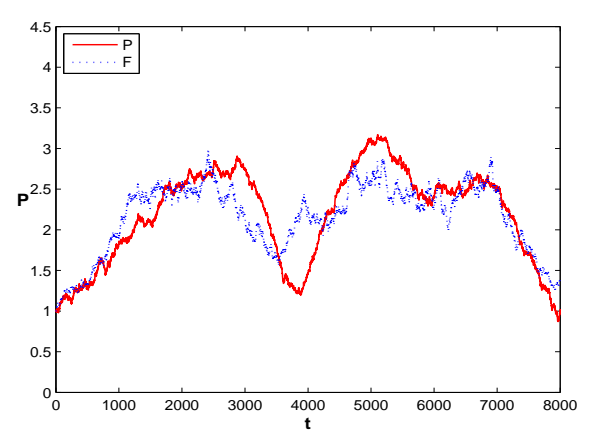

(a) Prices for $\tau=3$

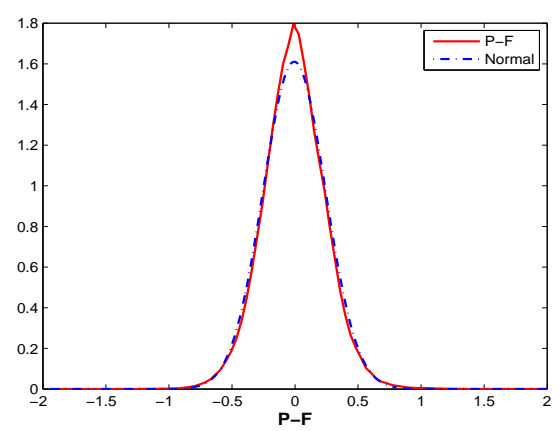

(c) Price deviation density for $\tau=3$

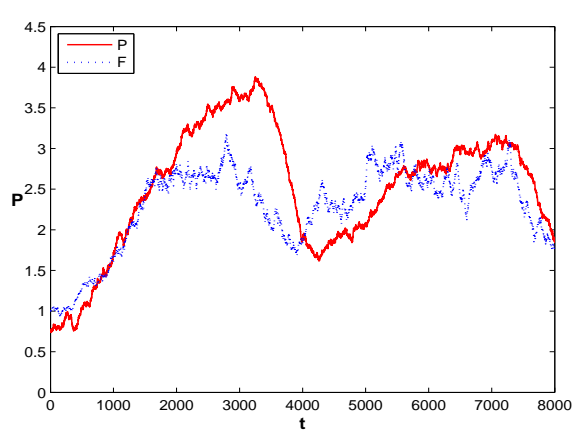

(b) Prices for $\tau=16$

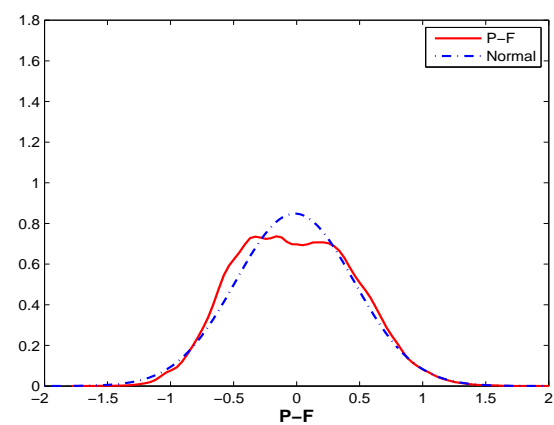

(d) Price deviation density for $\tau=16$

Figure D.1. The time series of the fundamental price $F(t)$ (the blue dotted line) and the market prices $P(t)$ (the red solid line) with (a) $\tau=3$ and (b) $\tau=16$, and the distributions of the deviations of the market prices from the fundamental prices $P(t)-F(t)$ with (c) $\tau=3$ and (d) $\tau=16$ for the HL model. Here $\sigma_{F}=0.12$ and $\sigma_{M}=0.15$. 


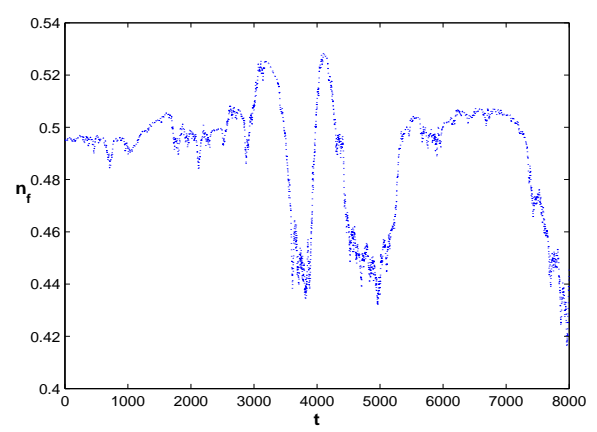

(a) Market fraction for $\tau=3$

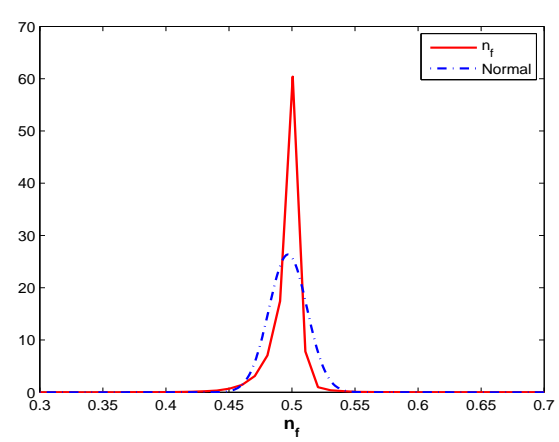

(c) Market fraction density for $\tau=3$

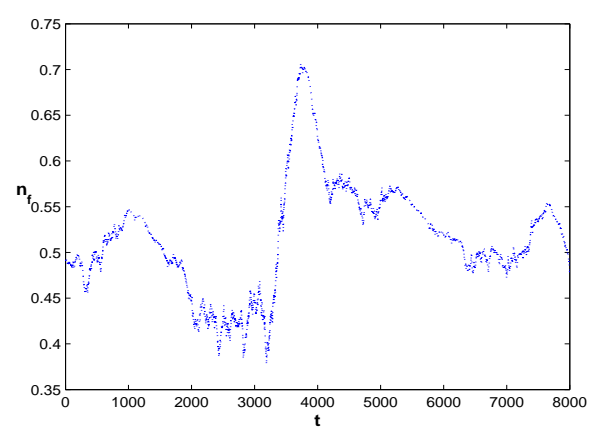

(b) Market fraction for $\tau=16$

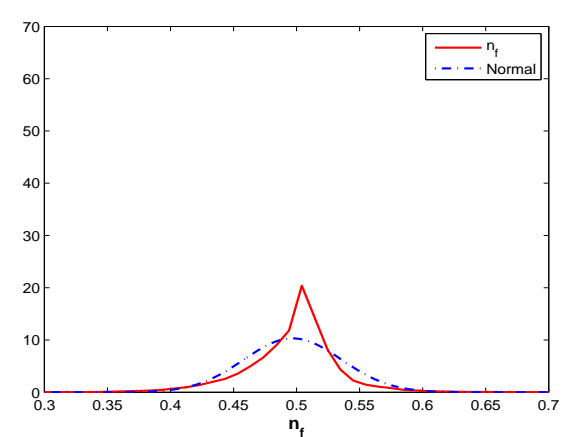

(d) Market fraction density for $\tau=16$

FigurE D.2. The time series of the fractions of the fundamentalists with (a) $\tau=3$ and (b) $\tau=16$ and the corresponding distributions with (c) $\tau=3$ and (d) $\tau=16$ for the HL model. Here $\sigma_{F}=0.12$ and $\sigma_{M}=0.15$. 


\section{Appendix E. Noises and the Stylized FaCts}

This appendix presents the time series properties and AC patterns of the return, absolute return, and squared return of the model with both the market and fundamental noises in Fig. E.1, the market noise only in Fig. E.2, and the fundamental noise only in Fig. E.3. 


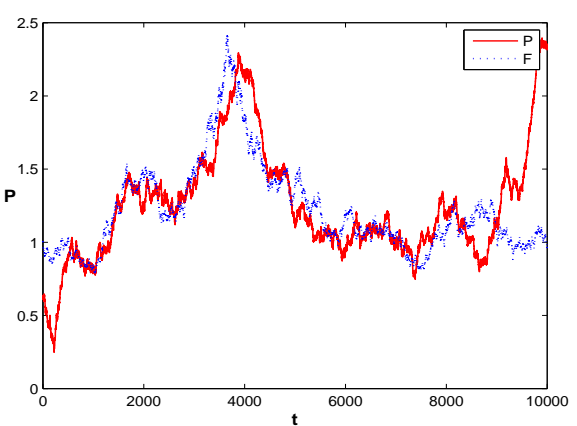

(a) The market price and the fundamental price

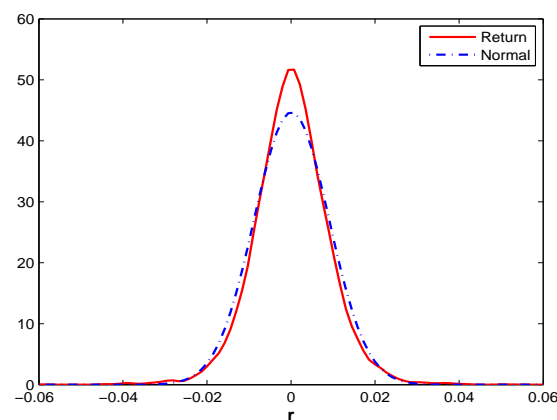

(c) The density of the market returns

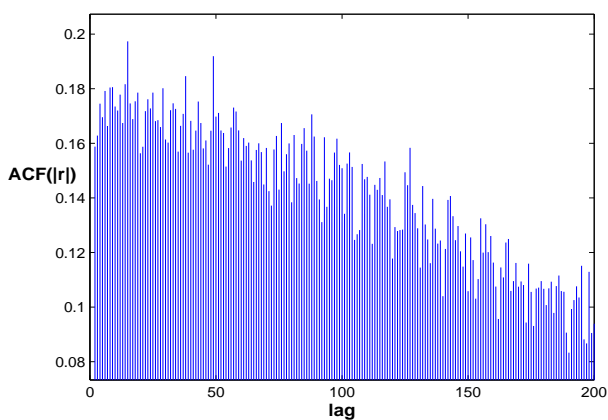

(e) The ACs of the absolute returns

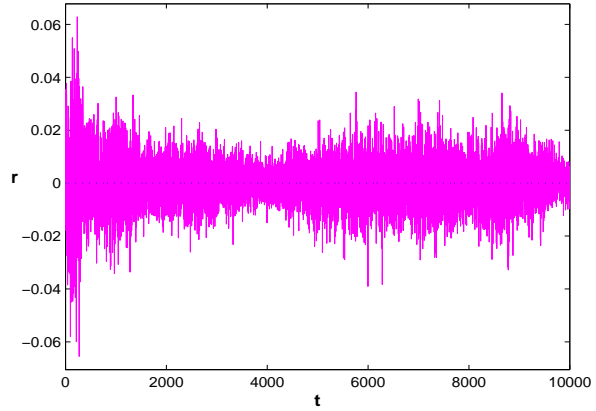

(b) The market returns $(r)$

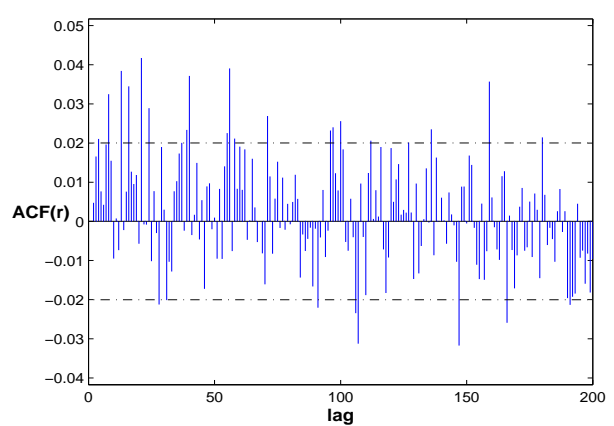

(d) The ACs of the market returns

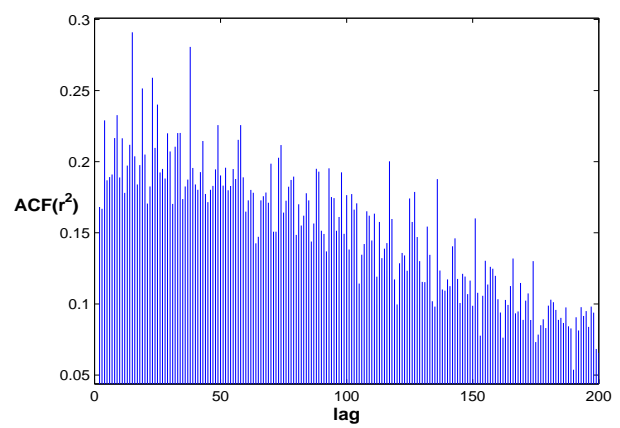

(f) The ACs of the squared returns

FigurE E.1. The effect of the two noises: the time series of (a) the market price (red solid line) and the fundamental price (blue dotted line) and (b) the market returns; (c) the return distribution; the ACs of (d) the returns; (e) the absolute returns, and (f) the squared returns. Here $\sigma_{F}=0.12$ and $\sigma_{M}=0.15$. 


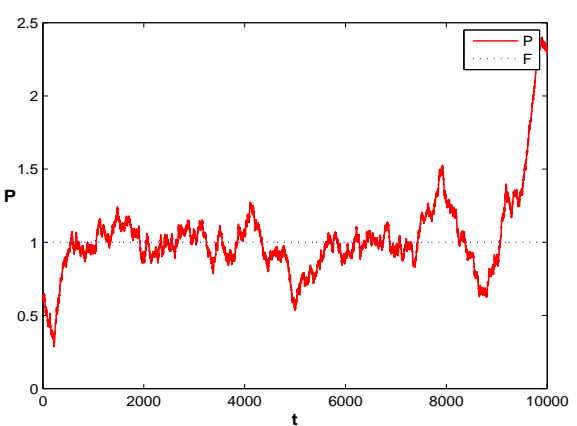

(a) The market price and the fundamental price

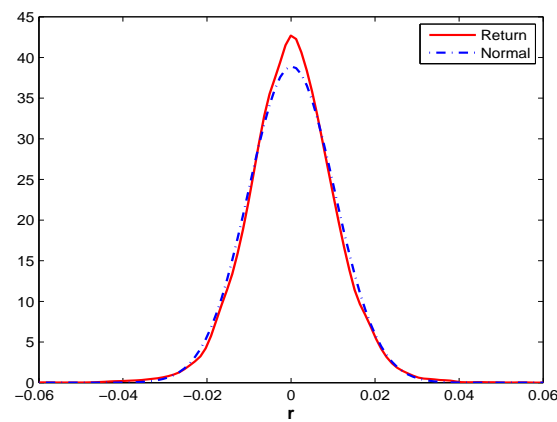

(c) The density of the market returns

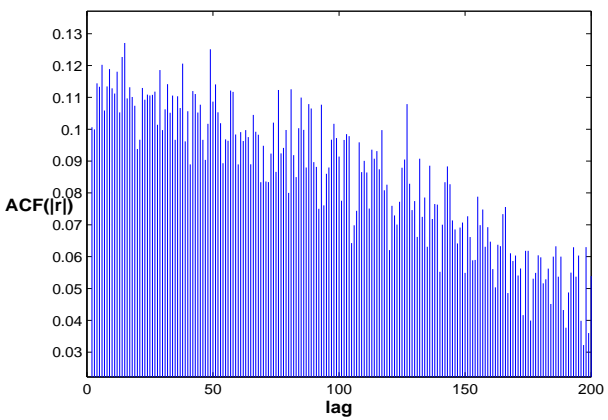

(e) The ACs of the absolute returns

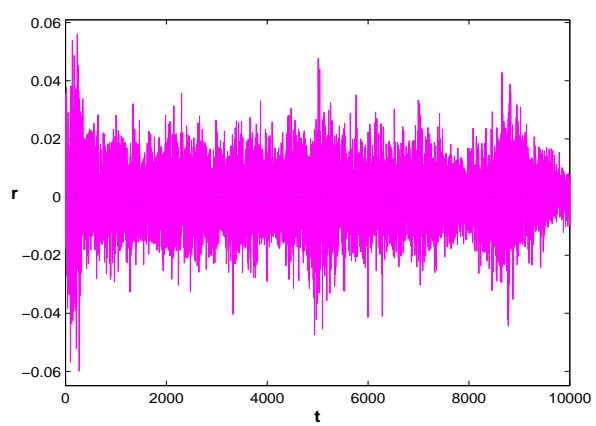

(b) The market returns $(r)$

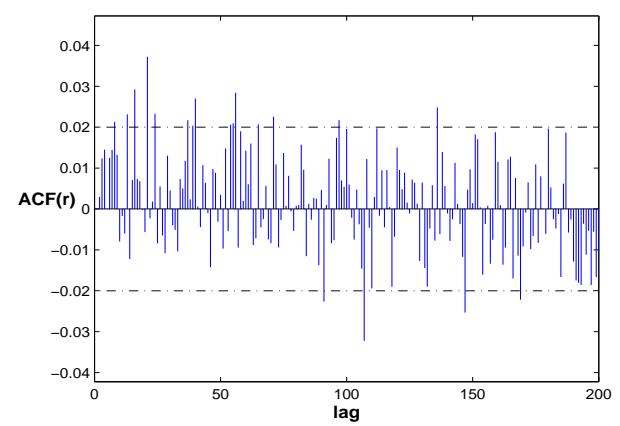

(d) The ACs of the market returns

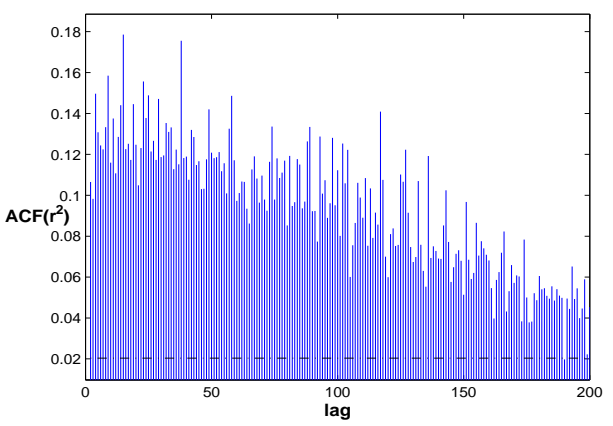

(f) The ACs of the squared returns

Figure E.2. The effect of the market noise only: the time series of (a) the market price (red solid line) and the fundamental price (blue dotted line) and (b) the market returns; (c) the return distribution; the ACs of (d) the returns; (e) the absolute returns, and (f) the squared returns. Here $\sigma_{F}=0$ and $\sigma_{M}=0.15$. 


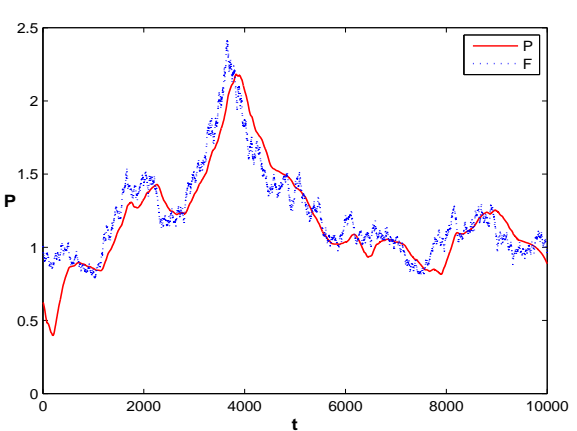

(a) The market price and the fundamental price

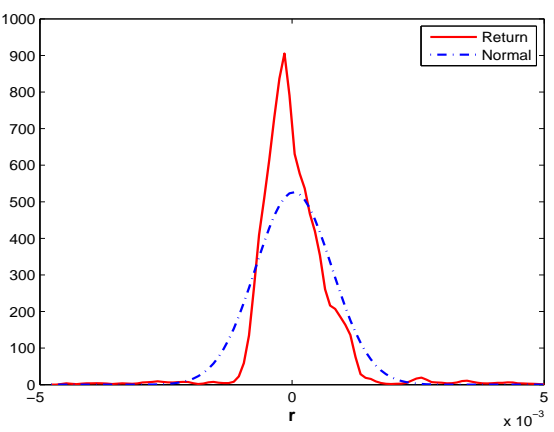

(c) The density of the market returns

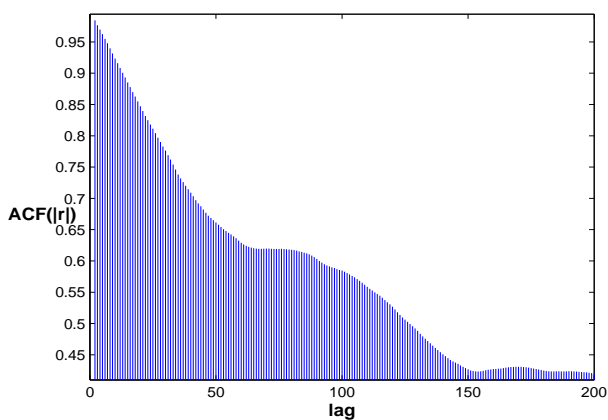

(e) The ACs of the absolute returns

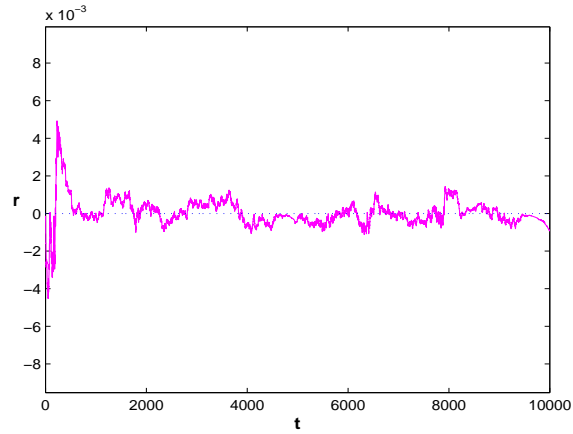

(b) The market returns $(r)$

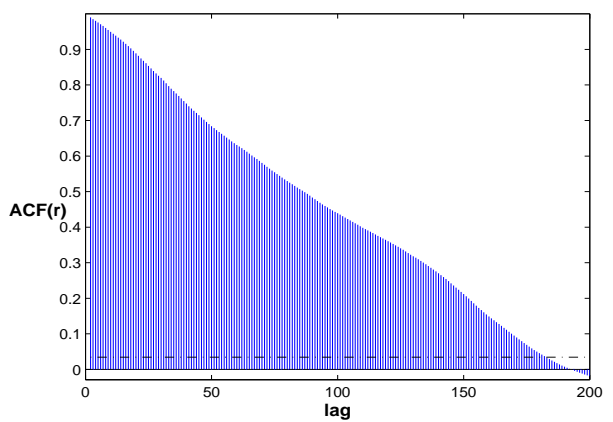

(d) The ACs of the market returns

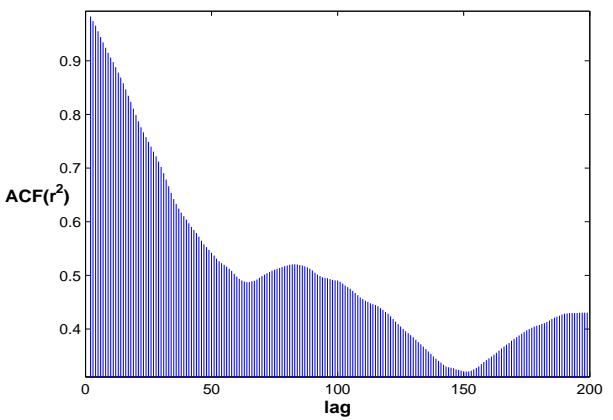

(f) The ACs of the squared returns

Figure E.3. The effect of the fundamental noise only: the time series of (a) the market price (red solid line) and the fundamental price (blue dotted line) and (b) the market returns; (c) the return distribution; the ACs of (d) the returns; (e) the absolute returns, and (f) the squared returns. Here $\sigma_{F}=0.12$ and $\sigma_{M}=0$. 


\section{REFERENCES}

Alfarano, S., Lux, T. and Wagner, F. (2005), 'Estimation of agent-based models: The case of an asymmetric herding model', Computational Economics 26, 19-49.

Alfarano, S., Lux, T. and Wagner, F. (2008), 'Time variation of higher moments in a financial market with heterogeneous agents: An analytical approach', Journal of Economic Dynamics and Control 32, 101-136.

Aoki, M. (2002), Modeling Aggregate Behaviour and Fluctuations in Economics, Cambridge University Press.

Banerjee, A. (1992), 'A simple model of herd behavior', Quarterly Journal of Economics 107, 797817.

Beja, A. and Goldman, M. (1980), 'On the dynamic behavior of prices in disequilibrium', Journal of Finance 35, 235-247.

Brock, W. and Hommes, C. (1997), 'A rational route to randomness', Econometrica 65, 1059-1095.

Brock, W. and Hommes, C. (1998), 'Heterogeneous beliefs and routes to chaos in a simple asset pricing model', Journal of Economic Dynamics and Control 22, 1235-1274.

Chen, S.-H., Chang, C. and Du, Y. R. (2012), 'Agent-based economic models and econometrics', Knowledge Engineering Review 27, 187-219.

Chiarella, C. (1992), 'The dynamics of speculative behaviour', Annals of Operations Research $37,101-123$.

Chiarella, C., Dieci, R. and He, X. (2009), Heterogeneity, Market Mechanisms and Asset Price Dynamics, Elsevier, pp. 277-344. in Handbook of Financial Markets: Dynamics and Evolution, Eds. Hens, T. and K.R. Schenk-Hoppe.

Chiarella, C., Dieci, R., He, X. and Li, K. (2013), 'An evolutionary CAPM under heterogeneous beliefs', Annals of Finance $\mathbf{9}, 185-215$.

Chiarella, C. and Di Guilmi, C. (2011a), 'The financial instability hypothesis: A stochastic microfoundation framework', Journal of Economic Dynamics and Control 35, 1151-1171.

Chiarella, C. and Di Guilmi, C. (2011b), Limit distribution of evolving strategies in financial markets, working paper 294, Quantitative Finance Research Centre, University of Technology, Sydney.

Chiarella, C. and He, X. (2002), 'Heterogeneous beliefs, risk and learning in a simple asset pricing model', Computational Economics 19, 95-132.

Chiarella, C. and He, X. (2003), 'Dynamics of beliefs and learning under $a_{l}$-processes - The Heterogeneous case', Journal of Economic Dynamics and Control 27, 503-531.

Chiarella, C., He, X. and Hommes, C. (2006), 'A dynamic analysis of moving average rules', Journal of Economic Dynamics and Control 30, 1729-1753. 
Franke, R. and Westerhoff, F. (2012), 'Structural stochastic volatility in asset pricing dynamics: Estimation and model contest', Journal of Economic Dynamics and Control 36, 1193-1211.

He, X. and Li, K. (2012), 'Heterogeneous beliefs and adaptive behaviour in a continuous-time asset price model', Journal of Economic Dynamics and Control 36, 973-987.

He, X. and Li, K. (2014), Time series momentum and market stability, working paper 341, Quantitative Finance Research Centre, University of Technology, Sydney.

He, X., Li, K. and Li, Y. (2014), 'Optimality of momentum and reversal', Working Paper .

He, X., Li, K., Wei, J. and Zheng, M. (2009), 'Market stability switches in a continuous-time financial market with heterogeneous beliefs', Economic Modelling 26, 1432-1442.

He, X. and Li, Y. (2007), 'Power law behaviour, heterogeneity, and trend chasing', Journal of Economic Dynamics and Control 31, 3396-3426.

He, X. and Zheng, M. (2010), 'Dynamics of moving average rules in a continuous-time financial market model', Journal of Economic Behavior and Organization 76, 615-634.

Hohnisch, M. and Westerhoff, F. (2008), 'Business cycle synchronization in a simple Keynesian macro-model with socially transmitted economic sentiment and international sentiment spillover', Structural Change and Economic Dynamics 19, 249-259.

Hommes, C. (2006), Heterogeneous Agent Models in Economics and Finance, Vol. 2 of Handbook of Computational Economics, North-Holland, pp. 1109-1186. in Agent-based Computational Economics, Eds. Tesfatsion, L. and K.L. Judd.

LeBaron, B. (2006), Agent-based Computational Finance, Vol. 2 of Handbook of Computational Economics, North-Holland, pp. 1187-1233. in Agent-based Computational Economics, Eds. Tesfatsion, L. and K.L. Judd.

Lux, T. (1995), 'Herd behaviour, bubbles and crashes', Economic Journal 105, 881-896.

Lux, T. (2009), Stochastic Behavioural Asset Pricing and Stylized Facts, Elsevier, pp. 161-215. in Handbook of Financial Markets: Dynamics and Evolution, Eds. Hens, T. and K.R. SchenkHoppé.

Moskowitz, T., Ooi, Y. H. and Pedersen, L. H. (2012), 'Time series momentum', Journal of Financial Economics 104, 228-250.

Scharfstein, D. and Stein, J. (1990), 'Herd behavior and investment', American Economic Review 80, 465-479. 